\title{
Breakdown study based on direct in situ observation of inner surfaces of an rf accelerating cavity during a high-gradient test
}

\author{
Tetsuo Abe, Tatsuya Kageyama, Hiroshi Sakai, Yasunao Takeuchi, and Kazuo Yoshino \\ Accelerator Laboratory, High Energy Accelerator Research Organization (KEK), \\ Tsukuba, Ibaraki 305-0801, Japan
}

(Received 1 September 2015; revised manuscript received 30 June 2016; published 4 October 2016)

\begin{abstract}
We have developed normal-conducting accelerating single-cell cavities with a complete higher-ordermode (HOM) heavily damped structure, into which we feed a 508.9-MHz continuous wave. During a highgradient test of the second production version of the cavity, we performed a breakdown study based on direct in situ observation of the inner surfaces of the cavity. This paper presents our experimental findings obtained from this observation.
\end{abstract}

DOI: 10.1103/PhysRevAccelBeams.19.102001

\section{INTRODUCTION}

Particle acceleration using radio-frequency (rf) electromagnetic waves is a widely used method in both large and small accelerators. Normal-conducting rf accelerating cavities (hereafter, $\mathrm{rf}$ cavities or cavities) are adequate and often used for high-current storage rings with beam currents of a few amperes or higher and high-gradient accelerators with gradients of several tens of megavolts per meter or higher, where high-purity copper is currently considered to be the best material for cavity bodies based on a comprehensive evaluation of the electric conductivity, thermal conductivity, outgassing rate, rf breakdown field, ease of machining, and cost. For the above-mentioned former (high-current) and latter (high-gradient) types of accelerators, rf cavities are required to have continuously high stability, i.e., an extremely low cavity breakdown rate over a long period of time, and a high gradient with a sufficiently-low cavity breakdown rate, respectively. To satisfy these requirements, the cavity breakdown rate should be minimized; however, this is currently very difficult because the real source of cavity breakdowns, i.e., the cavity-breakdown trigger mechanism, is unknown.

After fabricating rf cavities and before using them in accelerator operation, we perform a high-gradient test called rf conditioning, where we first feed a low rf power into the cavity, and gradually increase the input rf power and the accelerating field while maintaining certain cavityrelated quantities, such as the vacuum pressure (light blue lines in Fig. 7) and cavity breakdown rate [1], at values lower than their corresponding reference values.

\footnotetext{
tetsuo.abe@kek.jp
}

Published by the American Physical Society under the terms of the Creative Commons Attribution 3.0 License. Further distribution of this work must maintain attribution to the author(s) and the published article's title, journal citation, and DOI.
In the early stages of rf conditioning, cavity breakdowns occur frequently with increases in vacuum pressure and/or vacuum arcs, even when the accelerating field is low. This process removes contaminations and microscopic defects in the cavity surface using an rf field. Once the accelerating gradient reaches its maximum expected value in the highgradient test, almost no further increases in vacuum pressure occur, and the cavity breakdown rate usually decreases to an acceptable level at a gradient lower than the maximum achieved gradient unless the vacuum is broken or a long time passes without feeding a high rf power. Here rf cavities should be designed to ensure that the maximum expected gradient is sufficiently higher than the accelerator design requirement. However, the cavity breakdown rate rapidly increases as the accelerating gradient increases. Therefore, technology that enables a lower final cavity breakdown rate can contribute to developments of future accelerators with higher luminosities and/or higher energies.

Various experimental breakdown studies have been performed and have provided measurements of various quantities, such as the cavity breakdown rate and its dependence on the accelerating gradient, the rf waveform when cavity breakdown occurs, the dark current before and after cavity breakdown occurs and its dependence on the accelerating gradient, and partial or total vacuum pressure increases during high-gradient tests (e.g., [2-4]). In particular, a large amount of breakdown studies have been performed on normal-conducting $\mathrm{X}$-band accelerating structures using traveling or standing waves in pulsed operation, leading to the discovery of (1) a scaling law describing the cavity breakdown rate as a function of the gradient and rf pulse width (e.g., [5]); (2) the significant effect of the magnetic field and pulsed surface heating on the cavity breakdown rate [6,7]; and (3) a new local field quantity called the modified Poynting vector, which gives the high-gradient performance limit by cavity breakdown [5]. However, these measured quantities provide only indirect information about the cavity breakdown trigger, meaning it is difficult to use 
them to learn about the trigger. On another front, vacuum arc experiments using simple electrode setups, such as parallel plates and needle-plane electrodes, have been performed (e.g., [8-10]). We can learn a great deal about the breakdown characteristics of materials and surface processes from such experiments; however, there are significant differences between such setups and rf cavities regarding their electromagnetic field patterns and quality factors. In particular, rf cavities usually have a high quality factor of the order of thousands or higher for an accelerating field. After such high-gradient tests or vacuum arc experiments, microscopic inspections were

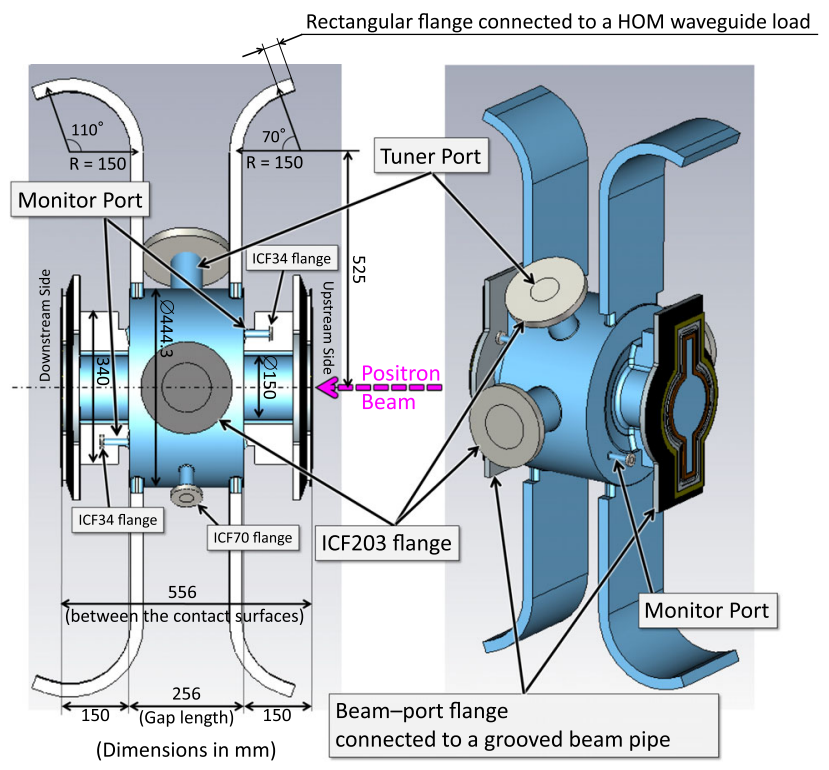

(a) Conceptual diagram of the DR cavity. The blue region is a vacuum during high-gradient operation. The gap length and diameter of this cavity are 256 and $444.3 \mathrm{~mm}$, respectively.

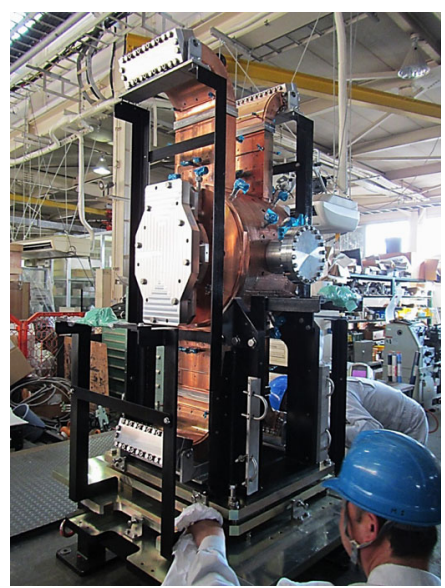

(b) DR cavity No. 2 immediately after its delivery.

FIG. 1. DR cavity. often performed, in which microscopic craters, cracks, and/or suspicious objects were observed on surfaces exposed to high electric and/or magnetic fields (e.g., $[10,11])$. These are assumed to originate from the breakdowns; however, it is unclear if we can find any association with the cavity breakdown trigger in any way.

To elucidate the cavity-breakdown trigger mechanism, we propose a method based on direct in situ observation of inner surfaces of an of cavity through video recording of light images during a high-gradient test. This method has the following three features: (1) Exhaustive observation, meaning all cavity breakdown events are captured; (2) Multidirectional and wide-field observation to allow the observation of the origin of the cavity breakdown; and (3) Accompanying statistical quantitative analysis of the data obtained from the observation. Hereafter, this method is called the direct observation method.

In this paper, through a breakdown study of an $\mathrm{rf}$ cavity developed for a positron damping ring (DR) at the SuperKEKB accelerator [12], we present our experimental findings obtained using the direct observation method, and demonstrate the usefulness of this method. Abbreviations used in this paper are listed in Table VII of Appendix A.

\section{EXPERIMENTAL SETUP AND METHODS}

\section{A. rf cavity for the DR (DR cavity)}

Figure 1(a) shows a conceptual diagram of the DR cavity, which is a pillbox cavity of a single cell used to excite a continuous $\mathrm{TM}_{010}$ standing wave as the accelerating mode. The accelerating voltage per cavity [cavity voltage $\left(V_{c}\right)$ ] during DR operation is $0.70 \mathrm{MV}$ and has an equivalent accelerating gradient $\left(E_{\mathrm{acc}}\right)$ of $2.7 \mathrm{MV} / \mathrm{m}$. This cavity voltage is comparable to actual operating voltages of other accelerating cavities of the

TABLE I. Design parameters of the DR cavity.

\begin{tabular}{lc}
\hline rf operating frequency & $508.9 \mathrm{MHz}$ \\
$R_{\text {sh }} / Q_{0}{ }^{\mathrm{a}}$ & $150 \Omega$ \\
$Q_{0}$ & $\approx 30000\left(97 \% \mathrm{IACS}^{\mathrm{b}}\right)$ \\
Input coupling factor & $\approx 1.3$ \\
Cavity voltage during operation & $V_{c}=0.70 \mathrm{MV}$ \\
& $\left(E_{\mathrm{acc}}=2.7 \mathrm{MV} / \mathrm{m}\right)$ \\
& $V_{c}=0.80 \mathrm{MV}$ \\
Cavity voltage of the specification & $\left(E_{\mathrm{acc}}=3.1 \mathrm{MV} / \mathrm{m}\right)$ \\
& $\approx 110 \mathrm{~kW}$ \\
Wall loss power at $V_{c}=0.70 \mathrm{MV}$ & $\approx 140 \mathrm{~kW}$ \\
Wall loss power at $V_{c}=0.80 \mathrm{MV}$ & 3.7 \\
$E_{s}^{(\max ) \mathrm{c}} / E_{\text {acc }}$ & $9.6 \mathrm{kAm}{ }^{-1}(\mathrm{MV} / \mathrm{m})^{-1}$ \\
$H_{s}^{(\max ) \mathrm{d}} / E_{\text {acc }}$ & \\
\hline \hline${ }^{\mathrm{a}} R_{\mathrm{sh}}$ and $Q_{0}$ represent the shunt impedance and unloaded \\
quality factor of the accelerating mode, respectively. \\
${ }^{\mathrm{b}} \mathrm{IACS}$ stands for International Annealed Copper Standard. \\
${ }^{\mathrm{c}} E_{s}^{(\max )}$ represents the maximum surface electric field. \\
${ }^{\mathrm{d}} H_{s}^{(\max )}$ represents the maximum surface magnetic field.
\end{tabular}


same type (normal-conducting single cells with continuous input waves in the ultrahigh frequency range) used in recent high-energy accelerator operations; for example, PEP-II (476 MHz) [13] was operated at 0.50 to $0.64 \mathrm{MV} /$ cavity [14], and the Australian Synchrotron (500 MHz) [15] has been operated at 0.60 to $0.75 \mathrm{MV} /$ cavity [16]. Typical design parameters of the DR cavity are summarized in Table I.

The main body of the DR cavity is made of high-purity oxygen-free copper (C10100, ASTM F68 Class 1), and consists of a barrel and two discoidal end plates. The inner surfaces of the barrel and the two end plates were machined with an accuracy of $50 \mu \mathrm{m}$ and a surface roughness of $3 \mathrm{~S}$ to $6 \mathrm{~S}$. Before the final brazing, the two end plates were

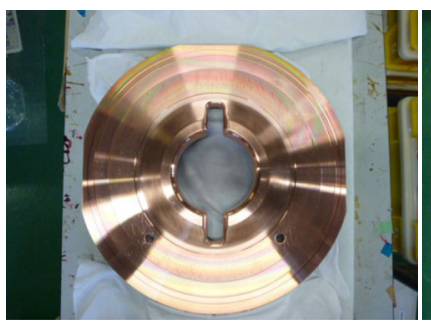

(a) Before electropolishing.

$R_{\mathrm{a}} \approx 1.5 \mu \mathrm{m}$. $R_{\mathrm{y}} \approx 8 \mu \mathrm{m}$.

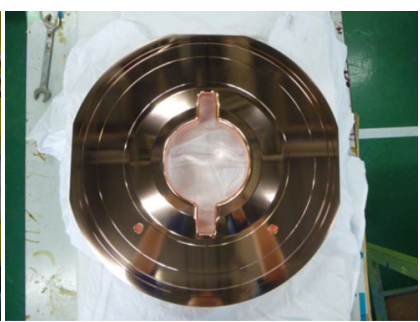

(b) After electropolishing. $R_{\mathrm{a}} \approx 0.2 \mu \mathrm{m}$. $R_{\mathrm{y}} \approx 1 \mu \mathrm{m}$.
FIG. 2. Photographs of the end plate (a) before and (b) after electropolishing with approximately $40 \mu \mathrm{m}$ of etching. The outer diameter is approximately $500 \mathrm{~mm}$. The inner diameter of the beam port is $150 \mathrm{~mm}$. electropolished; the barrel part was not electropolished. This process of electropolishing typically improves the surface roughness; in this case, the arithmetic mean roughness $\left(R_{\mathrm{a}}\right)$ and maximum peak roughness $\left(R_{\mathrm{y}}\right)$ were changed from 1.5 and $8 \mu \mathrm{m}$ to 0.2 and $1 \mu \mathrm{m}$ (typical measurements), respectively, as shown in Fig. 2, where the skin depth of copper at $508.9 \mathrm{MHz}$ is approximately $3 \mu \mathrm{m}$. We have demonstrated definite positive results of this type of electropolishing through high-gradient tests [17]. In the final brazing, the barrel and two end plates were bonded to form a pillbox cavity.

For the purpose of frequency tuning, one of the end plates has a bump (tuning bump) with an initial height of $2.5 \mathrm{~mm}$ in the surface region where a high magnetic field is applied during high-gradient operation (see Fig. 8). Reducing the bump height by $1.0 \mathrm{~mm}$ decreases the accelerating-mode frequency by $286 \mathrm{kHz}$. The end plates with and without such tuning bump are called the tuning end plate (TEP) and fixed end plate (FEP) in this paper, respectively. The FEP and TEP are located upstream and downstream of the barrel, respectively, with respect to the positron beam direction in the DR tunnel.

Further details of the DR cavity are given in Appendix B.

Thus far, we have fabricated two production versions of the DR cavity, the first (DR cavity No. 1) in fiscal year 2012 and the second (DR cavity No. 2) in fiscal year 2013, based on the development results of a prototype cavity (DR cavity No. 0) fabricated in fiscal year 2011 and tested early in fiscal year 2012. DR cavities No. 1 and No. 2 are based on the same electrical design, have the same mechanical

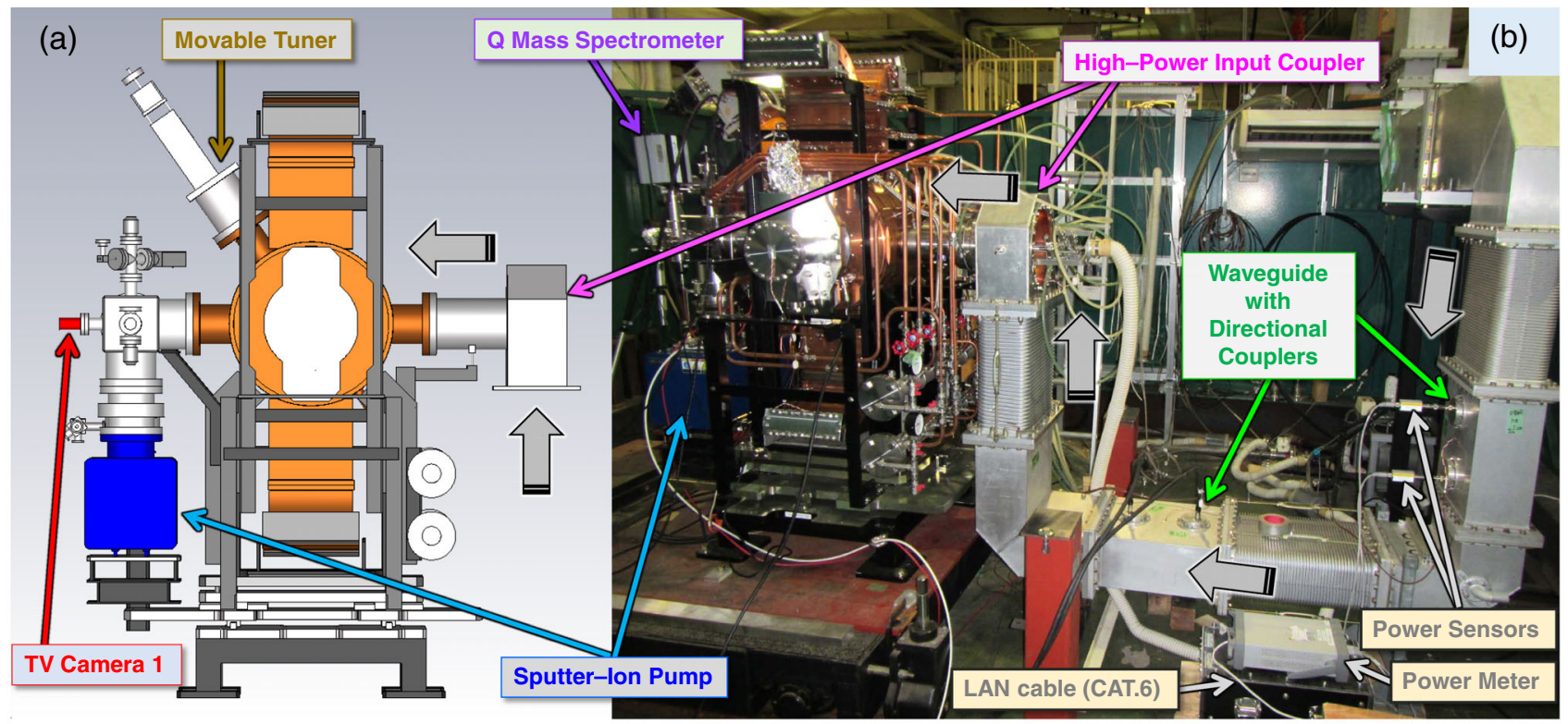

FIG. 3. (a) Diagram of DR cavity No. 2. (b) Photograph of the test stand used for the high-gradient test of DR cavity No. 2 in a radiation-controlled area. The bold arrows indicate the flow of high rf power generated by a 1.2-MW continuous-wave klystron. The klystron is not included in this photograph. The turbo-molecular pump is behind the sputter-ion pump. 
structure, and were made using the same fabrication method. Figure 1(b) is a photograph of DR cavity No. 2 immediately after its delivery. This paper presents results of a breakdown study of this cavity through high-gradient testing.

\section{B. Test stand}

The setup of the high-gradient test for DR cavity No. 2 is shown in Fig. 3. We used a 1.2-MW continuous-wave klystron as a high-power source together with a four-port circulator to protect this klystron. High rf power was fed into the cavity through a coaxial-type high-power input coupler. The input coupler has fine grooves on the outer conductor of the coaxial line to suppress multipactoring [18]. The coupling factor of the input coupler was set to 1.3 at low rf power by adjusting its coupling loop angle.

A vacuum evacuation system was directly attached to the main body of the cavity; this system consists of two vacuum pumps located near the cavity: a turbo-molecular pump $(300 \mathrm{~L} / \mathrm{s})$ and a sputter-ion pump $(400 \mathrm{~L} / \mathrm{s})$.

The flow rate and temperature of the cooling water for the main body of the cavity were approximately $130 \mathrm{~L} / \mathrm{min}$ and $30^{\circ} \mathrm{C}$, respectively.

To implement the direct observation method, we fabricated two vacuum chambers containing mirrors (mirror chambers) and used them in this high-gradient test, as shown in Fig. 4. Near the beam axis, the radiation level is high during high-gradient operation; therefore, a mirror is located inside the mirror chamber to allow the observation of the inside of the cavity through the beam port. We attached a television (TV) camera with a charge-coupled device sensor to the view port of the mirror chamber far from the beam axis, as shown in Fig. 4(c). For DR cavity No. 1, we attached only one mirror chamber, which contained a circular mirror, to the upstream beam port [17], whereas for DR cavity No. 2, we attached two mirror chambers, each of which contained an elliptical mirror, to the upstream and downstream beam ports to obtain a wider

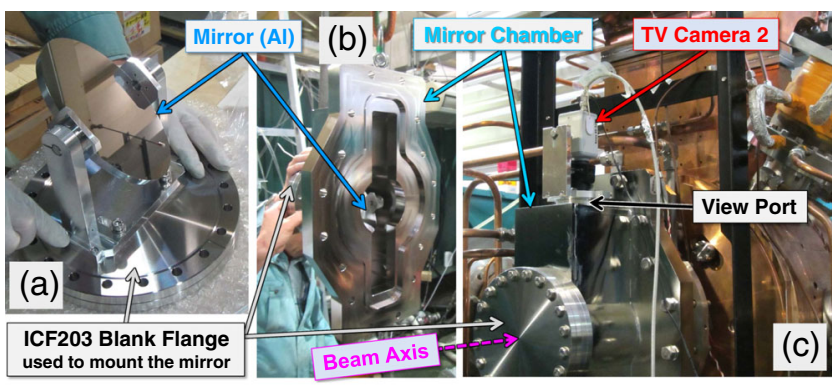

FIG. 4. Mirror chamber used for the high-gradient test of DR cavity No. 2. (a) Aluminum elliptical mirror mounted on an ICF203 blank flange. The surface was finished by lapping, yielding $R_{\mathrm{a}} \approx 1 \mathrm{~nm}$. (b) Stainless steel vacuum chamber containing the mirror. The inner surface of this chamber was electropolished. (c) Mirror chamber attached to DR cavity No. 2.

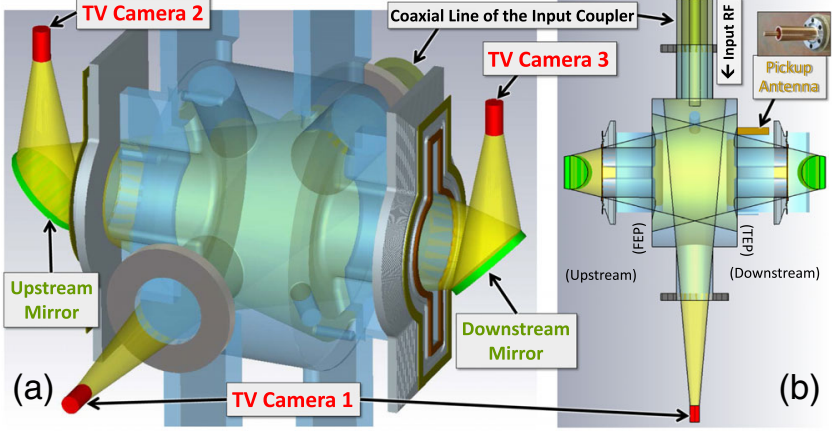

FIG. 5. (a) Schematic of the experimental devices. Red cylinders and yellow regions represent the three TV cameras and their fields of view, respectively. During the high-gradient test, the inside of the cavity (blue) was a vacuum, and the mirrors (green) were contained in the mirror chambers, as shown in Fig. 4. (b) Cross section along the horizontal plane including the center of the cavity.

view. In total, we used three TV cameras for the multidirectional and wide-field observation of the inside of the cavity, as shown in Fig. 5. TV camera 1 was attached to the viewport of the vacuum evacuation system on the side

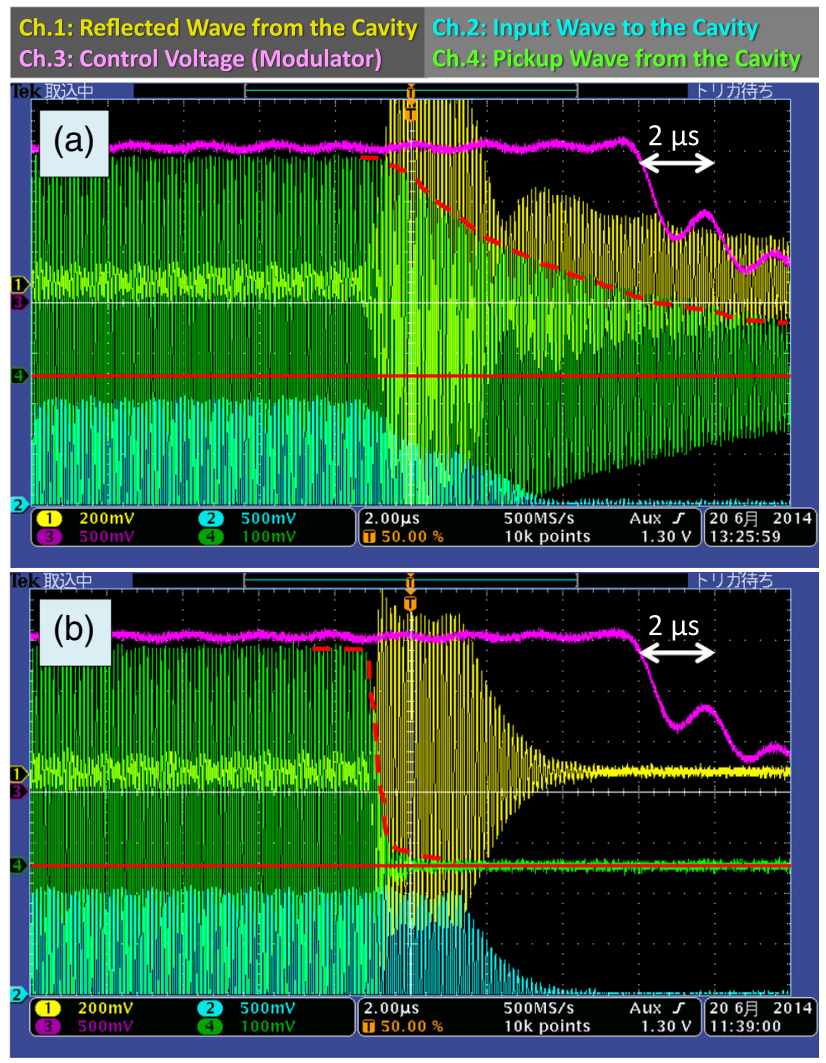

FIG. 6. Waveforms of the oscilloscope displayed for a time span of $20 \mu \mathrm{s}(=2 \mu \mathrm{s} /$ div $)$ when the interlock system was activated. The red dashed curves show the envelope of the 508.9-MHz pickup signal from DR cavity No. 2, and the red solid lines show the zero level. (a) Waveforms when the rf switch was turned off for a reason related to the klystron. (b) Example of cavity breakdown events. 
opposite the input coupler. TV camera 2 was attached to the upstream beam port and aimed toward the downstream end plate (the TEP). TV camera 3 was attached to the downstream beam port and aimed toward the upstream end plate (the FEP). Note that we use the words "upstream" and "downstream" for convenience; no beam was injected into the cavity during this high-gradient test.

Videos obtained using the TV cameras were automatically recorded on high-capacity storage devices using skip back recorders during the time period from $5 \mathrm{~s}$ before the rf switch of the low-level control system was changed from $\mathrm{ON}$ to $\mathrm{OFF}$, which is the recording trigger signal, until $1 \mathrm{~s}$ after this change. Additionally, we recorded the nonbreakdown status manually as needed. The videos were obtained and recorded with a frame rate of approximately 30 frames per second (fps) (more accurately, $29.97 \mathrm{fps}$ ) and an image resolution of $704 \times 480$ pixels, and videos with different trigger timings were stored in different electronic files, each of which had a file size of approximately $13 \mathrm{MB}$ in MOV format. This exhaustive observation enables the statistical quantitative data analysis performed in this study.

\section{Detection of cavity breakdown}

In this study, cavity breakdown was detected when the interlock system was activated by an rf power reflected from the cavity exceeding a specified threshold, and in addition, the signal through the pickup antenna from the cavity (pickup signal) decayed much more rapidly than the decay time constant (approximately $8 \mu \mathrm{s}$ ) determined by the loaded quality factor (approximately 13000) and frequency. Here, such threshold was in the range of 16 to $28 \mathrm{~kW}$ depending on the actual reflected rf power $\left(\lesssim 3 \mathrm{~kW}\right.$ for $\left.V_{c} \leq 0.95 \mathrm{MV}\right)$. Figure 6(a) shows an example of events that occurred when the interlock system was activated for a reason unrelated to the cavity, where such decay time constant was observed in the pickup signal. Conversely, Fig. 6(b) shows an example of the cavity breakdown events in which the pickup signal dropped in less than $1 \mu \mathrm{s}$.

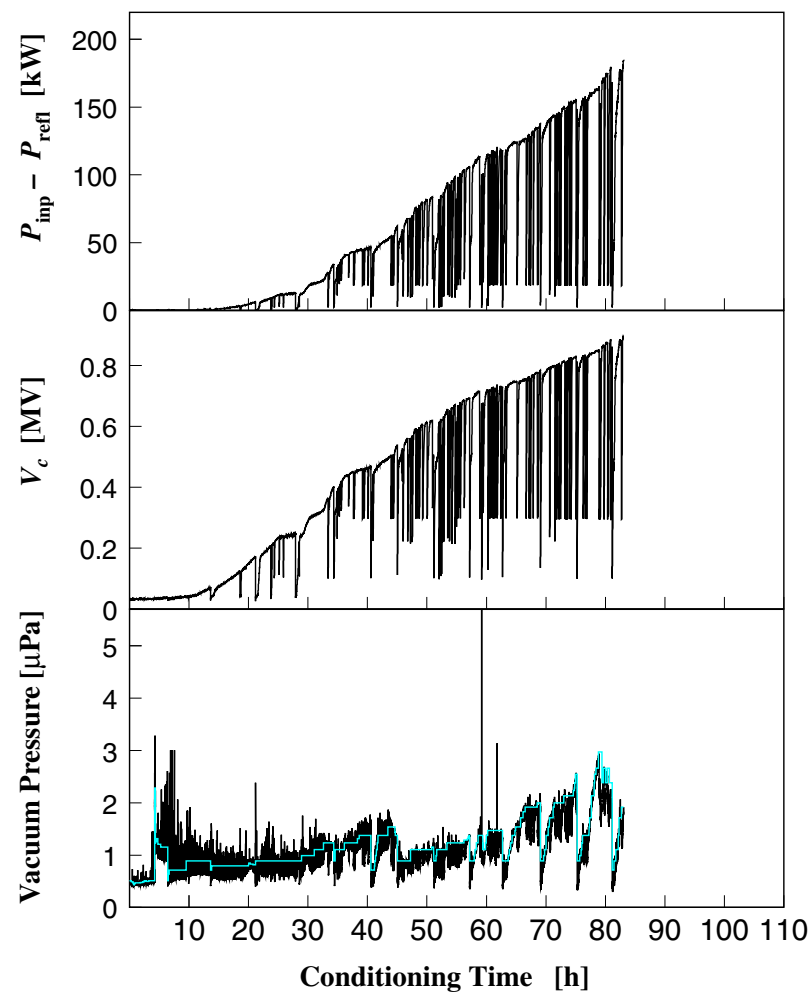

(a) DR cavity No. 1.

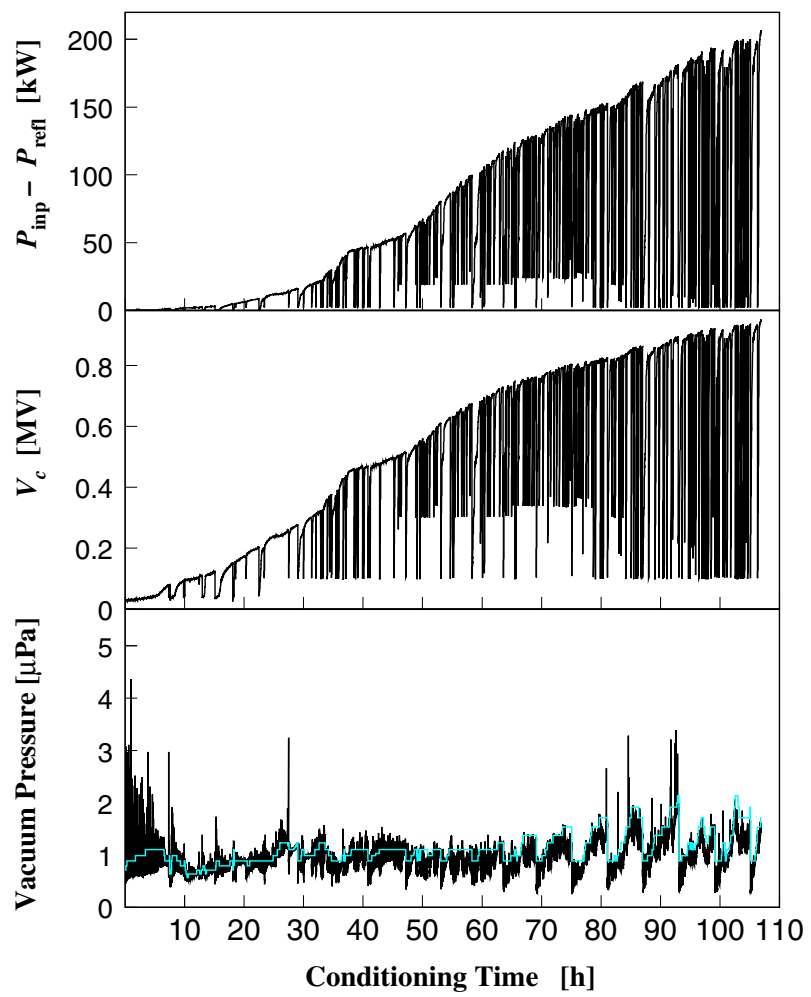

(b) DR cavity No. 2.

FIG. 7. Conditioning histories until $V_{c}$ reached (a) $0.90 \mathrm{MV}$ for DR cavity No. 1 and (b) 0.95 MV for DR cavity No. 2 with data points recorded every $10 \mathrm{~s}$. Data with no input rf power, data while the input rf power was kept constant, and data used to tune the control system or klystron are excluded here. The light blue lines indicate the reference vacuum pressure specified in the automatic conditioning by computer control. If the vacuum pressure is higher than the reference pressure, the rf power is stepped down until the vacuum pressure becomes lower than the reference pressure, and then the power is gradually stepped up as long as the vacuum pressure is lower than the reference pressure. The step-up slope is proportional to the difference between the reference and vacuum pressures. 

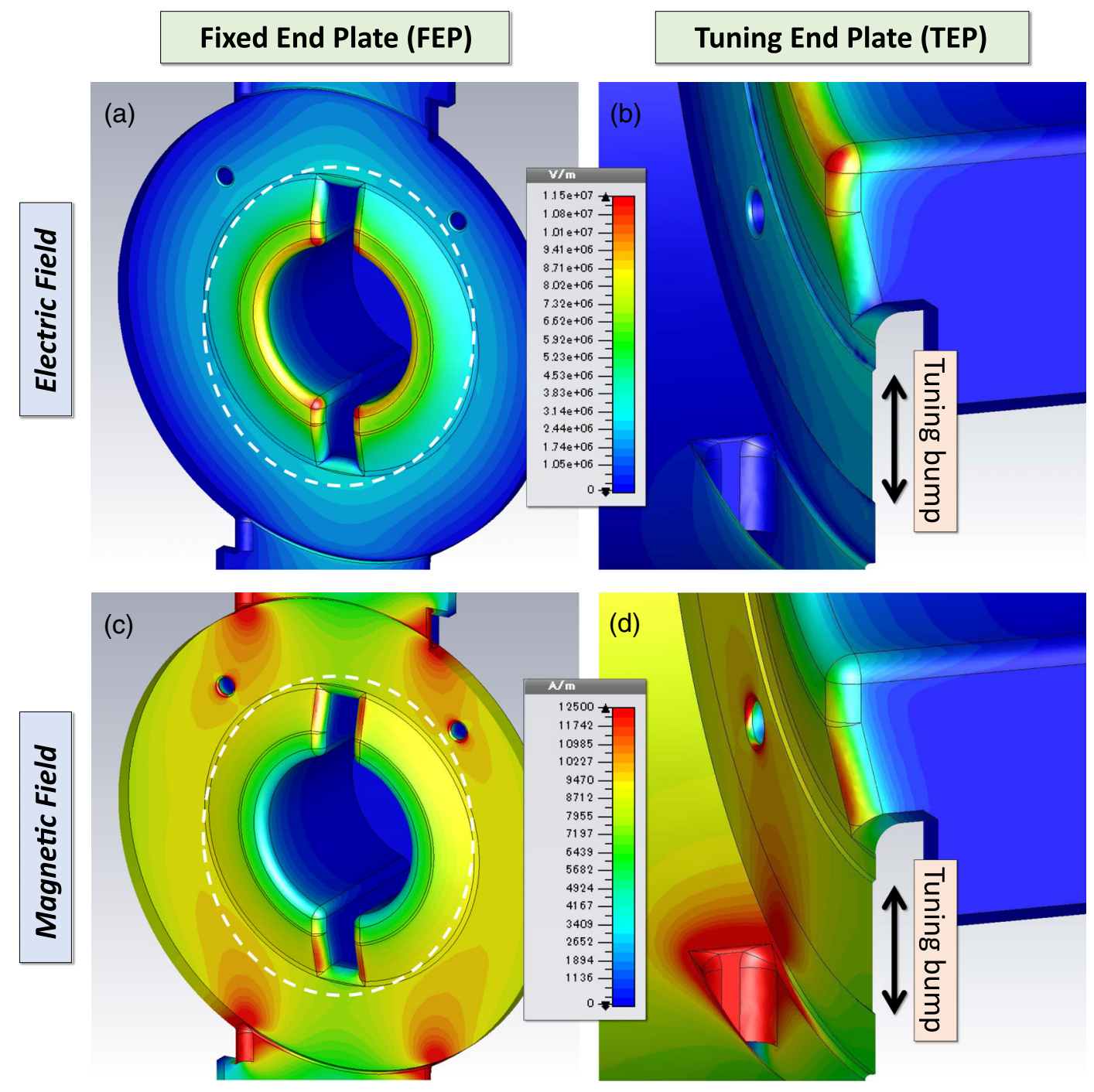

FIG. 8. Surface field of the accelerating mode at $V_{c}=0.90 \mathrm{MV}\left(E_{\mathrm{acc}}=3.5 \mathrm{MV} / \mathrm{m}\right)$. The region inside the white dashed ellipse on the FEP was observable with TV camera 3, and the observable region on the TEP was almost the same. The maximum strengths of the electric and magnetic fields are $13 \mathrm{MV} / \mathrm{m}$ on the nose cone and $34 \mathrm{kA} / \mathrm{m}$ at the aperture for the HOM waveguide, respectively. (a) Electric field on the FEP. (b) Electric field on the TEP with a vertical cut plane. (c) Magnetic field on the FEP. (d) Magnetic field on the TEP with a vertical cut plane.

After detecting cavity breakdown, the rf switch was automatically turned off, and the acquired data, including the rf waveforms and vacuum pressure, were then manually reviewed over a period typically spanning 30-60 s. The

TABLE II. Classification and number of cavity trip events.

\begin{tabular}{lccc}
\hline \hline Period & $\mathrm{Vac}^{\mathrm{a}}$ & $\mathrm{BD}^{\mathrm{b}}$ & Others \\
\hline rf conditioning & 2 & 180 & 1 \\
Stability test (total) & 0 & 25 & 0 \\
Stability test $\left(V_{c}=0.90 \mathrm{MV}\right)$ & 0 & 11 & 0 \\
\hline \hline
\end{tabular}

${ }^{\mathrm{a}}$ Vac means cavity vacuum events.

${ }^{\mathrm{b}} \mathrm{BD}$ means cavity breakdown events. automatic conditioning by computer control was then manually restarted from zero rf power if no abnormality was found. The algorithm of the automatic conditioning is described in the caption of Fig. 7.

TABLE III. Number of cavity breakdown events with an abnormality observed by at least one of the three TV cameras. Numbers enclosed in square brackets are percentages of the total numbers of cavity breakdown events with binomial errors for a $1 \sigma$ confidence interval.

\begin{tabular}{lc}
\hline \hline Period & $\begin{array}{c}\text { Events with an } \\
\text { abnormality observed }\end{array}$ \\
\hline rf conditioning & $143[79 \pm 3 \%]$ \\
Stability test (total) & $20\left[80_{-11}^{+8} \%\right]$ \\
Stability test $\left(V_{c}=0.90 \mathrm{MV}\right)$ & $9\left[82_{-19}^{+12} \%\right]$ \\
\hline \hline
\end{tabular}


TABLE IV. Classification of the cavity breakdown events according to the phenomenon observed by the TV cameras at the moment of cavity breakdown. Numbers enclosed in square brackets are percentages of the total numbers of cavity breakdown events with binomial errors for a $1 \sigma$ confidence interval. Examples of cavity breakdown events classified as the "spot-type explosion of a stable bright spot", "spot-type explosion not originating from a stable bright spot", "non-spot-type flash", and "non-spot-type lightning only" columns are shown in Figs. 9, 12, 16(b), and 16(c) and (d), respectively. Detailed definitions of these event types are given in Sec. IVA.

\begin{tabular}{|c|c|c|c|c|c|}
\hline Period & $\begin{array}{c}\text { Spot-type explosion } \\
\text { of a stable } \\
\text { bright spot }\end{array}$ & $\begin{array}{l}\text { Spot-type explosion } \\
\text { not originating from a } \\
\text { stable bright spot }\end{array}$ & $\begin{array}{l}\text { Non-spot-type } \\
\text { flash }\end{array}$ & $\begin{array}{l}\text { Non-spot-type } \\
\text { lightning only }\end{array}$ & Others \\
\hline rf conditioning & $\begin{array}{c}44 \\
{[24 \pm 3 \%]}\end{array}$ & $\begin{array}{c}72 \\
{[40 \pm 4 \%]}\end{array}$ & $\begin{array}{c}4 \\
{[2 \pm 1 \%]}\end{array}$ & $\begin{array}{c}14 \\
{[8 \pm 2 \%]}\end{array}$ & $\begin{array}{c}9 \\
{[5 \pm 2 \%]}\end{array}$ \\
\hline Stability test (total) & {$\left[28_{-10}^{+12} \%\right]$} & $\begin{array}{c}9 \\
{\left[36_{-11}^{+12} \%\right]}\end{array}$ & {$\left[\begin{array}{c}3 \\
{\left[12_{-6}^{+10} \%\right.}\end{array}\right.$} & {$\left[\begin{array}{c}1 \\
{\left[4_{-3}^{+7} \%\right]}\end{array}\right.$} & $\begin{array}{c}0 \\
{\left[0_{-0}^{+7} \%\right]}\end{array}$ \\
\hline $\begin{array}{l}\text { Stability test } \\
\left(V_{c}=0.90 \mathrm{MV}\right)\end{array}$ & {$\left[\begin{array}{c}5 \\
\left.45_{-18}^{+19} \%\right]\end{array}\right.$} & {$\left[\begin{array}{c}2 \\
{\left[18_{-12}^{+19} \%\right.}\end{array}\right.$} & {$\left[\begin{array}{c}1 \\
9_{-8}^{+16} \%\end{array}\right]$} & {$\left[\begin{array}{c}1 \\
9_{-8}^{+16} \%\end{array}\right]$} & {$\left[\begin{array}{c}0 \\
0 \\
0.0\end{array}\right]$} \\
\hline
\end{tabular}

\section{Calculation of cavity voltage $V_{c}$}

In this study, $V_{c}$ was calculated from the rf power input to the cavity $\left(P_{\text {inp }}\right)$, the rf power reflected from the cavity $\left(P_{\text {refl }}\right)$, and the quantities given in Table $\mathrm{I}$, according to the formulae described in $[12,17]$. Both of these rf powers $\left(P_{\text {inp }}\right.$ and $\left.P_{\text {refl }}\right)$ were measured using a power meter and sensors connected directly to the directional coupler near the cavity, as shown in Fig. 3(b).

\section{HIGH-GRADIENT TEST}

We first gradually increased the rf power input to DR cavity No. 2 using an automatic computer control to a

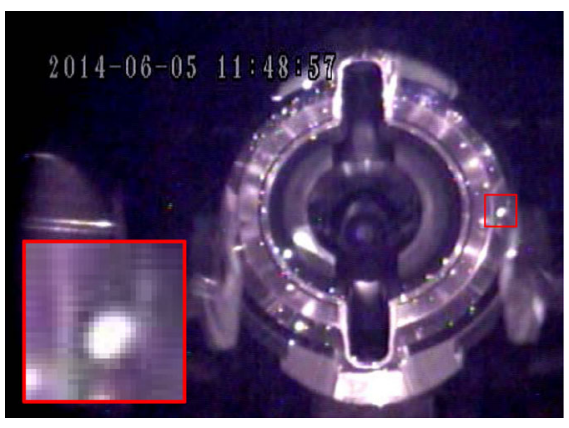

(a) 1 frame $(1 / 30 \mathrm{~s})$ before this cavity breakdown.

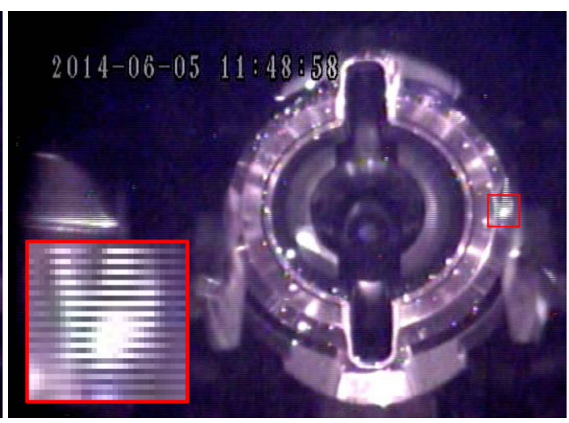

(b) At the moment of this cavity breakdown.

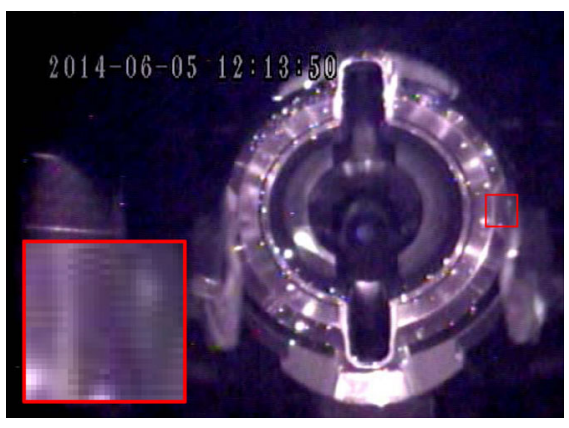

(c) Shortly after recovering from this cavity breakdown at $V_{c}=0.95 \mathrm{MV}$.

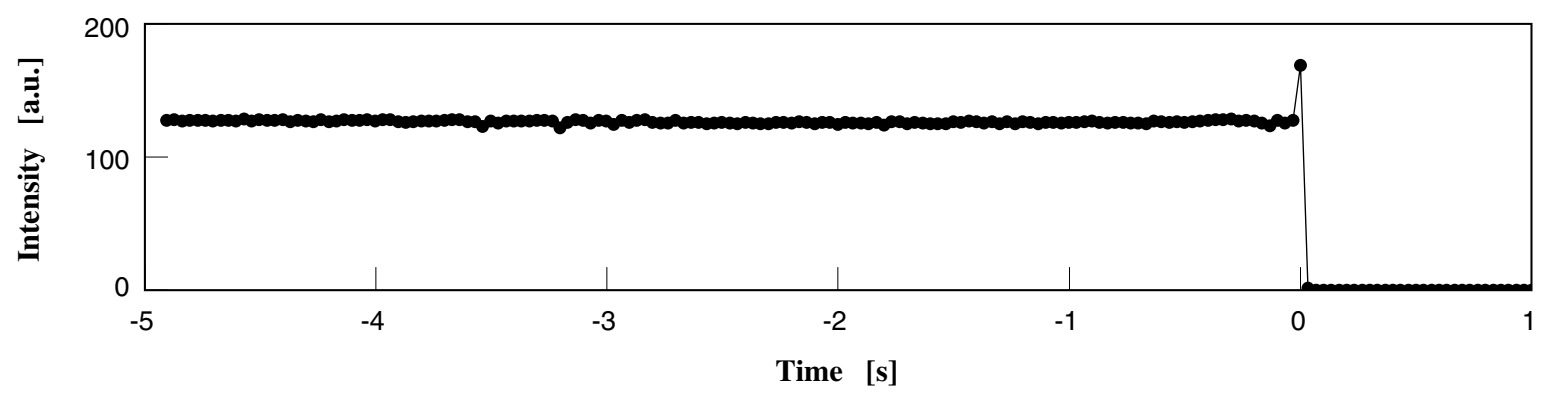

(d) Intensity of the pixels in the region inside the red square for each frame during the time period from $5 \mathrm{~s}$ before until $1 \mathrm{~s}$ after this cavity breakdown. The origin of the horizontal axis is the time at which this cavity breakdown occurred.

FIG. 9. Example of spot-type explosion events with a stable bright spot. (a), (b), and (c) are snapshots, in chronological order, of the FEP recorded with TV camera 3 during the rf conditioning. The insets are magnified views of the region outlined by a red square. The bright spot at 3 o'clock [inside the red square in (a)] exploded at $V_{c}=0.95 \mathrm{MV}$, as shown in (b), and then disappeared, as shown in (c). A clear spike was observed in the intensity of the region outlined by a red square at the moment of this cavity breakdown, as shown in (d). 
TABLE V. Number of spot-type explosion events with a stable bright spot in which the bright spot disappeared after the explosion. Numbers enclosed in square brackets are percentages of the total numbers of cavity breakdown events with binomial errors for a $1 \sigma$ confidence interval.

\begin{tabular}{lc}
\hline \hline Period & $\mathrm{BS}^{\mathrm{a}}$ disappeared \\
\hline rf conditioning & 36 \\
Stability test (total) & {$[20 \pm 3 \%]$} \\
& 7 \\
Stability test $\left(V_{c}=0.90 \mathrm{MV}\right)$ & {$\left[28_{-10}^{+12} \%\right]$} \\
& 5 \\
\hline \hline
\end{tabular}

${ }^{\mathrm{a}} \mathrm{BS}$ indicates bright spot.

cavity voltage of $V_{c}=0.95 \mathrm{MV}$, which is the highest voltage permitted by the radiation regulation for this test stand, while maintaining the vacuum pressure at a value lower than the specified value, i.e., the reference vacuum pressure indicated by light blue lines in Fig. 7. Then, after reaching $V_{c}=0.95 \mathrm{MV}$, we performed a stability test at a constant cavity voltage of $V_{c}=0.90 \mathrm{MV}$. The former step is hereafter referred to as rf conditioning (performed May 12 to June 6, 2014, during the daytime on weekdays), and the latter is referred to as the stability test (performed June 10 to July 4, 2014, during the daytime on weekdays, excluding some maintenance days).

\section{A. rf conditioning}

Figure 7(b) shows the rf conditioning history of DR cavity No. 2, where $V_{c}$ reached $0.90 \mathrm{MV}(0.95 \mathrm{MV})$ in 95 (107) $\mathrm{h}$ without any problem, exceeding the cavity voltage of $0.80 \mathrm{MV}$ given in the specifications.

Figure 7(a) shows the rf conditioning history of DR cavity No. 1, which was obtained in the same manner as that for DR cavity No. 2. In this case, $V_{c}$ reached $0.90 \mathrm{MV}$ in $83 \mathrm{~h}$. Only data with $V_{c} \leq 0.90 \mathrm{MV}$ are plotted in Fig. 7(a) because data with $V_{c}>0.90 \mathrm{MV}$ are included in the stability test period for DR cavity No. 1. The

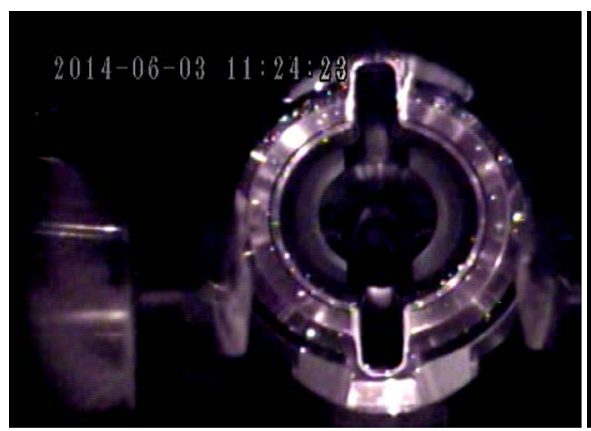

(a) During rf conditioning (first reaching $V_{c}=0.90 \mathrm{MV}$ )

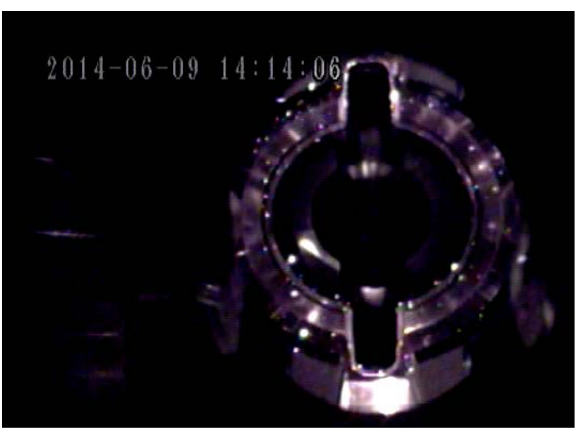

(b) One day before starting the stability test.

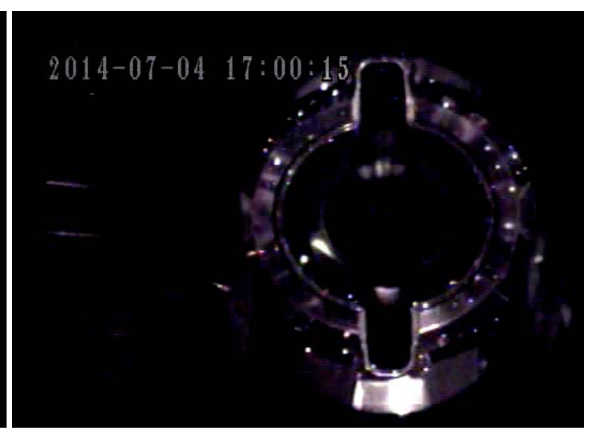

(c) $5 \mathrm{~s}$ before the end of this high-gradient test.

FIG. 10. Snapshots of the FEP recorded with TV camera 3 at $V_{c}=0.90 \mathrm{MV}$.

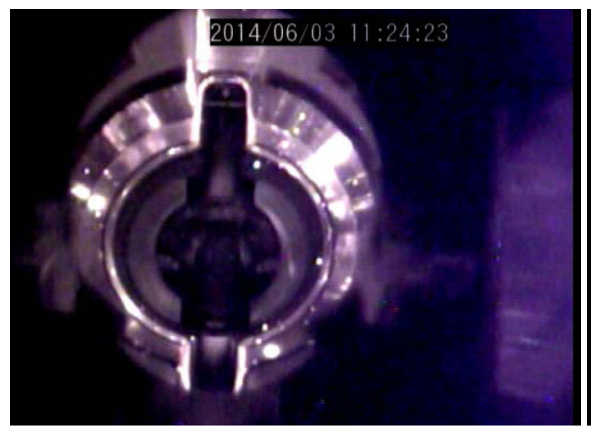

(a) During rf conditioning (first reaching $\left.V_{c}=0.90 \mathrm{MV}\right)$

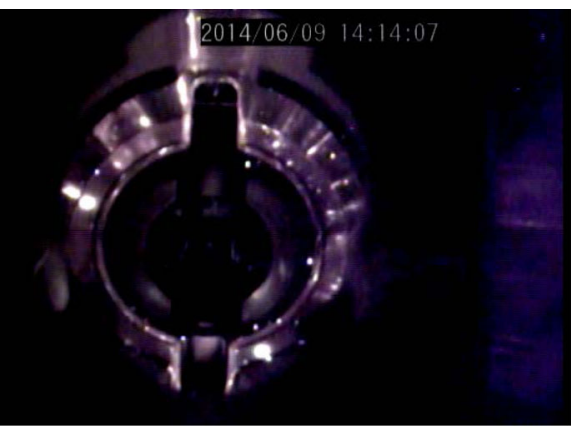

(b) One day before starting the stability test.

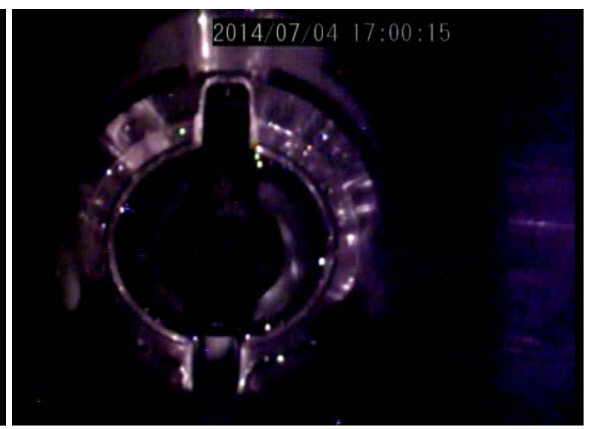

(c) $5 \mathrm{~s}$ before the end of this high-gradient test.

FIG. 11. Snapshots of the TEP recorded with TV camera 2 at $V_{c}=0.90 \mathrm{MV}$. 
conditioning speed of DR cavity No. 2 is comparable to that of DR cavity No. 1.

\section{B. Stability test}

After the rf conditioning, we performed a stability test on DR cavity No. 2 for 16 days according to the following daily sequential procedure. (1) The devices were turned on, and the cavity voltage was increased to 0.94 or $0.95 \mathrm{MV}$ in the morning. (2) This cavity voltage was maintained for $30 \mathrm{~min}$. (3) The cavity voltage was decreased to $V_{c}=0.90 \mathrm{MV}$. (4) The cavity voltage was maintained at $V_{c}=0.90 \mathrm{MV}$ until 17:00 that evening. Finally, we maintained a cavity voltage of $V_{c}=0.90 \mathrm{MV}$ for a total of $80 \mathrm{~h}$. Throughout this time, 11 cavity breakdowns occurred. This number of cavity breakdowns is equivalent to a cavity breakdown rate of $3.3_{-1.0}^{+1.3}$ breakdowns per day for DR cavity No. 2, where the statistical error for a $1 \sigma(68 \%)$ confidence interval is given according to Poisson statistics. This cavity breakdown rate at $V_{c}=0.90 \mathrm{MV}$ is statistically equivalent to that of DR cavity No. 1: $5.0_{-2.7}^{+4.8}$ breakdowns per day, which was obtained in the same manner [17].

\section{Bright spots during high-gradient operation}

As shown in Figs. 10 and 11, clear bright spots were observed on the end plates during high-gradient operation [19]. These bright spots maintained their intensity for hours or longer, and had no significant effects on the highgradient operation as long as they remained stable. As shown in Figs. 12(c) and 14(f), any bright spot did not continue without rf power for more than a few tens of milliseconds. Currently, the origin of the bright spots is unknown; however, it is presumed that they could be caused by microscopic protrusions on the copper surface or small particles from outside of the cavity adhering to the copper surface. To reveal their origin, more information is required, including the spectra of the bright spots. It is also noteworthy that neither the density nor the intensity of the bright spots was found to have any clear correlation with the surface field strength (Fig. 8).

\section{DATA ANALYSIS}

DR cavities No. 1 and No. 2 do not significantly differ in the following respects: (i) Unloaded quality factor [20],

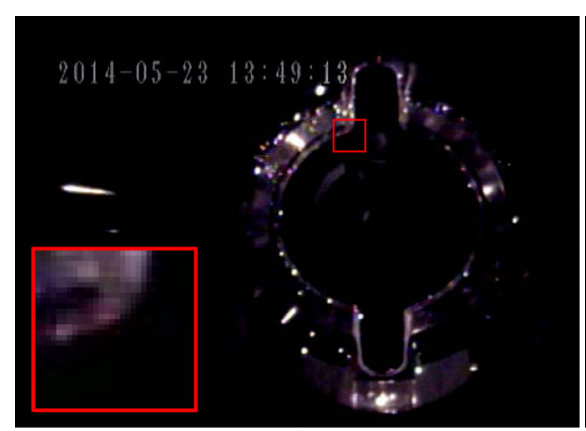

(a) 1 frame $(1 / 30 \mathrm{~s})$ before this cavity breakdown.

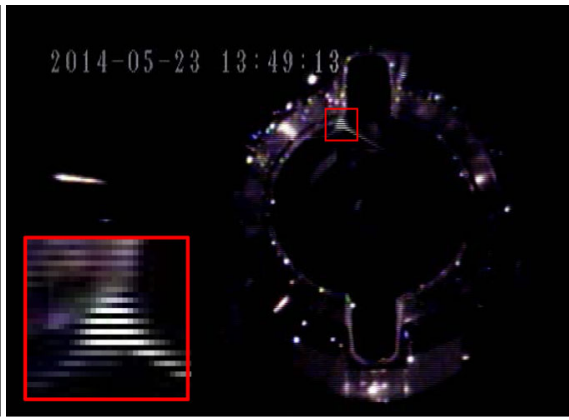

(b) At the moment of this cavity breakdown.

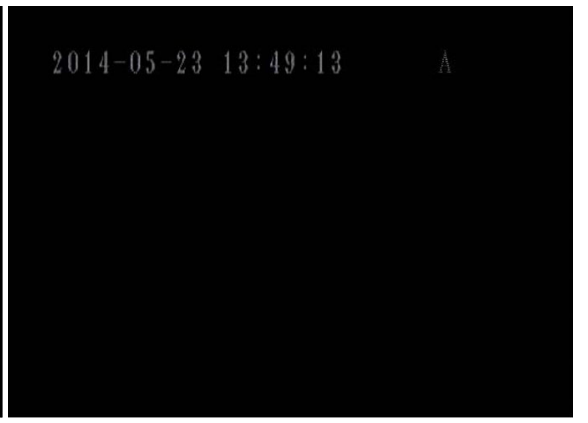

(c) 1 frame $(1 / 30 \mathrm{~s})$ after this cavity breakdown.

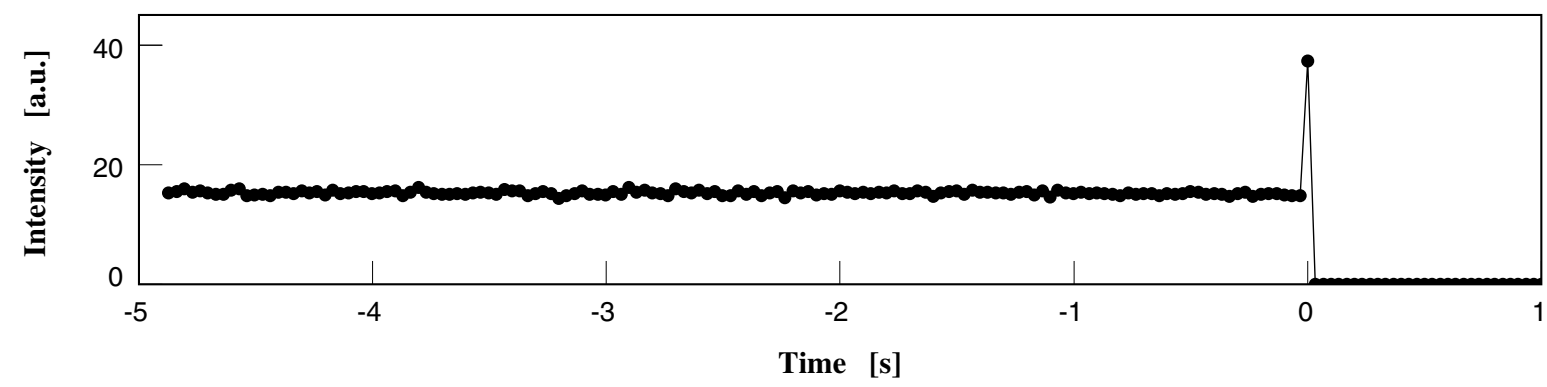

(d) Intensity of the pixels in the region inside the red square for each frame during the time period from $5 \mathrm{~s}$ before until $1 \mathrm{~s}$ after this cavity breakdown. The origin of the horizontal axis is the time at which this cavity breakdown occurred.

FIG. 12. Example of spot-type explosion events without a stable bright spot. (a), (b), and (c) are snapshots, in chronological order, of the FEP recorded with TV camera 3 during the rf conditioning. The insets are magnified views of the region outlined by a red square. A spot-type explosion occurred at 11 o'clock [inside the red square in (b)] at $V_{c}=0.65 \mathrm{MV}$; however, no bright spot was present in this region until one frame before this cavity breakdown, as indicated by the red square in (a). A clear spike was observed in the intensity of the region outlined by a red square at the moment of this cavity breakdown, as shown in (d). 
(ii) rf conditioning speed (Fig. 7), (iii) Vacuum performance (Fig. 7 and Appendix B), (iv) High-gradient performance (Fig. 7) and cavity breakdown rate (Sec. III B), and (v) Radiation dose rate or dark current [Fig. 19(a)]. Thus, DR cavity No. 2 has no particular problems or unusual characteristics. This means that performing a breakdown study on only DR cavity No. 2 does not result in a loss of generality.

\section{A. Classification and statistics of cavity breakdown events}

In this paper, a cavity trip is defined as an event during high-gradient operation when the interlock system is activated for a reason related to the cavity. Cavity trip events are classified into three types: (1) Cavity vacuum events, which are defined as events accompanied by a vacuum-pressure rise beyond a given threshold $\left(4.1 \times 10^{-5}\right.$ to $1.0 \times 10^{-4} \mathrm{~Pa}$ ), (2) Cavity breakdown events (defined in Sec. II C), and (3) Others. It should be noted that cavity breakdown events accompanied by an increase in the vacuum pressure beyond the threshold are not defined as cavity vacuum events but as cavity breakdown events. Table II shows the classification and number of cavity trip
TABLE VI. Number of cavity breakdown events accompanied by the sudden appearance of a new bright spot immediately before the cavity breakdown. Numbers enclosed in square brackets are percentages of the total numbers of cavity breakdown events with binomial errors for a $1 \sigma$ confidence interval.

\begin{tabular}{lc}
\hline \hline Period & Sudden $\mathrm{BS}^{\mathrm{a}}$ appearance \\
\hline rf conditioning & 6 \\
Stability test (total) & {$[3 \pm 1 \%]$} \\
& 0 \\
Stability test $\left(V_{c}=0.90 \mathrm{MV}\right)$ & {$\left[0_{-0}^{+7} \%\right]$} \\
& 0 \\
\hline \hline
\end{tabular}

${ }^{\mathrm{a}} \mathrm{BS}$ indicates bright spot.

events; almost all of these events were cavity breakdown events.

Table III gives the number of cavity breakdown events with an abnormality observed by at least one of the three TV cameras at the moment when the interlock system was activated, and the percentages in all the cavity breakdown events. These numbers and percentages were obtained by carefully reviewing every frame of every video.

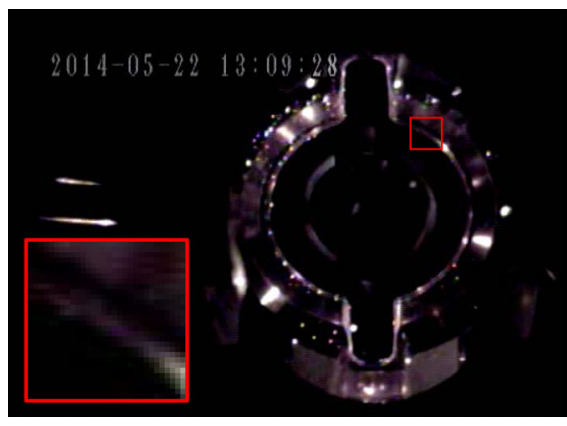

(a) 2 frames $(2 / 30 \mathrm{~s})$ before this cavity breakdown.

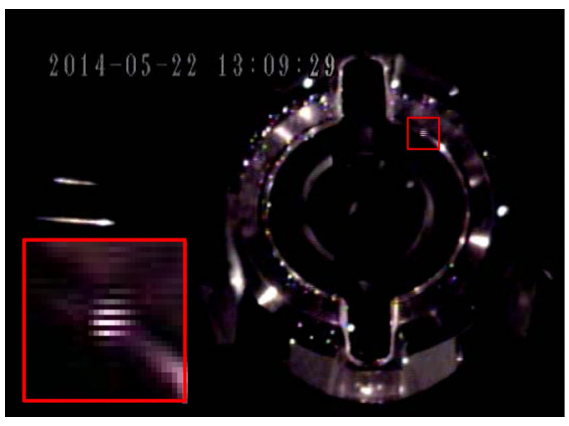

(b) 1 frame $(1 / 30 \mathrm{~s})$ before this cavity breakdown.

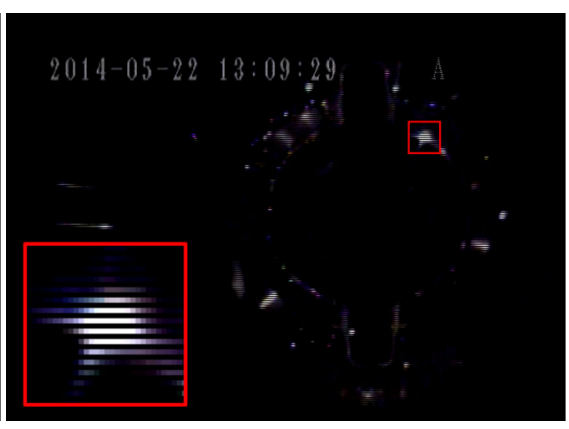

(c) At the moment of this cavity breakdown.

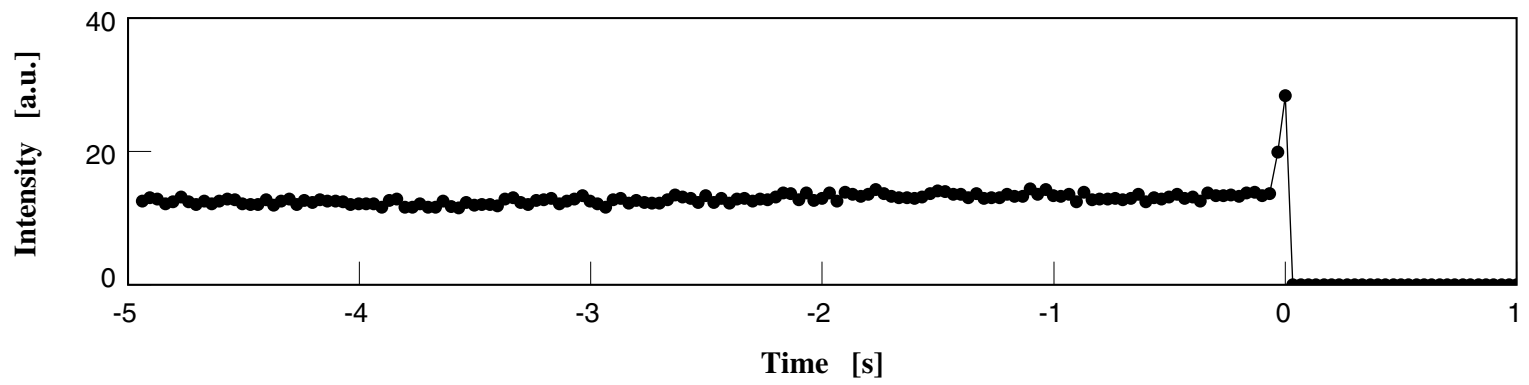

(d) Intensity of the pixels in the region inside the red square for each frame during the time period from $5 \mathrm{~s}$ before until $1 \mathrm{~s}$ after this cavity breakdown. The origin of the horizontal axis is the time at which this cavity breakdown occurred.

FIG. 13. Example of spot-type explosion events without a stable bright spot, accompanied by the sudden appearance of a new bright spot. (a), (b), and (c) are snapshots, in chronological order, of the FEP recorded with TV camera 3 during the rf conditioning. The insets are magnified views of the region outlined by a red square. There was an explosion at 1 o'clock [inside the red squares in (b) and (c)] at $V_{c}=0.56 \mathrm{MV}$; however, no bright spot was present in this region until 2 frames $(2 / 30 \mathrm{~s})$ before this cavity breakdown, as indicated by the red square in (a). A clear spike was observed in the intensity of the region outlined by a red square at the moment of this cavity breakdown for two frames, as shown in (d). 
Approximately $80 \%$ of all cavity breakdown events were accompanied by an abnormality during both the rf conditioning and the stability test; this large percentage means that most of the cavity breakdown events emit a signal that is observable by at least one of the TV cameras.

Table IV gives the classification of the cavity breakdown events, where an event classified as the "spot-type explosion of a stable bright spot" column (hereafter, a spot-type explosion event with a stable bright spot) is defined as a cavity breakdown event accompanied by a rapid increase in the intensity of a stable bright spot at the moment of the cavity breakdown. Figure 9 shows an example of such type of events, in which one of the stable bright spots on the FEP exploded and then disappeared.

Table $\mathrm{V}$ gives the number of cavity breakdown events accompanied by a bright spot explosion followed by the

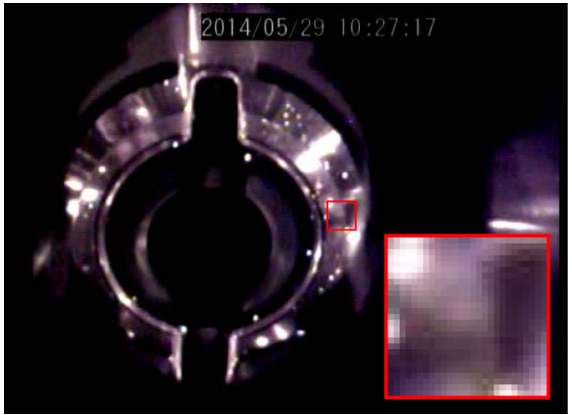

(a) 4 frames (4/30 s) before this cavity breakdown.

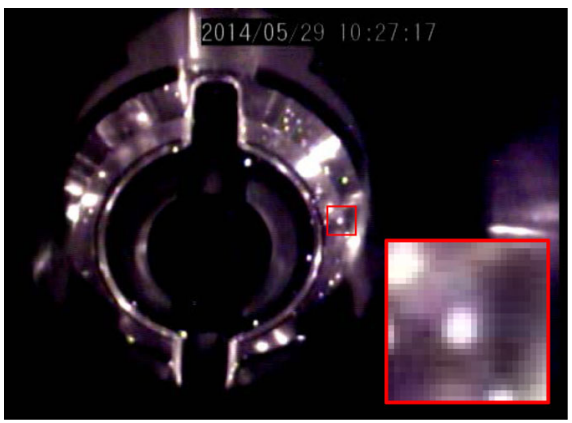

(d) 1 frame $(1 / 30 \mathrm{~s})$ before this cavity breakdown.

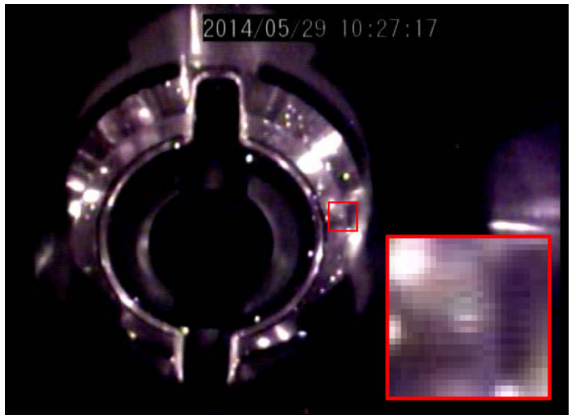

(b) 3 frames $(3 / 30 \mathrm{~s})$ before this cavity breakdown.

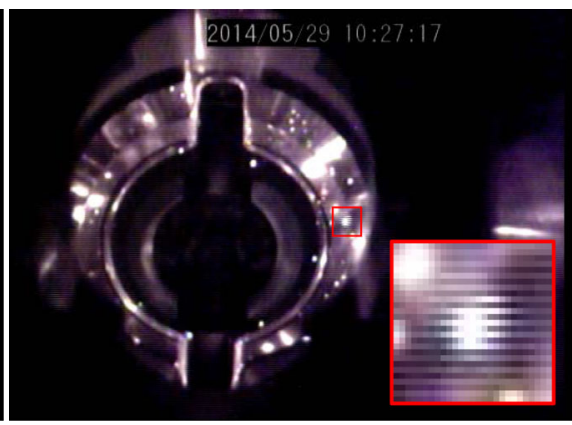

(e) At the moment of this cavity breakdown.

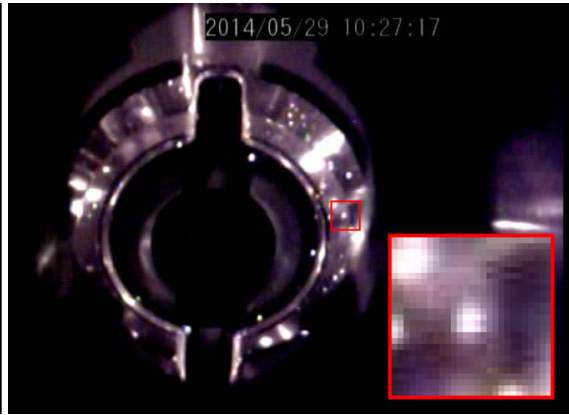

(c) 2 frames $(2 / 30 \mathrm{~s})$ before this cavity breakdown.

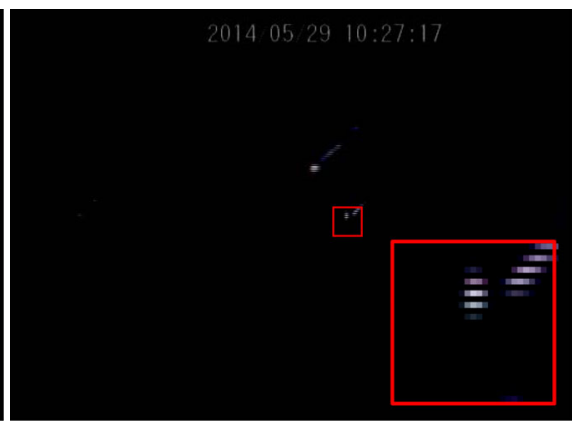

(f) 1 frame $(1 / 30 \mathrm{~s})$ after this cavity breakdown.

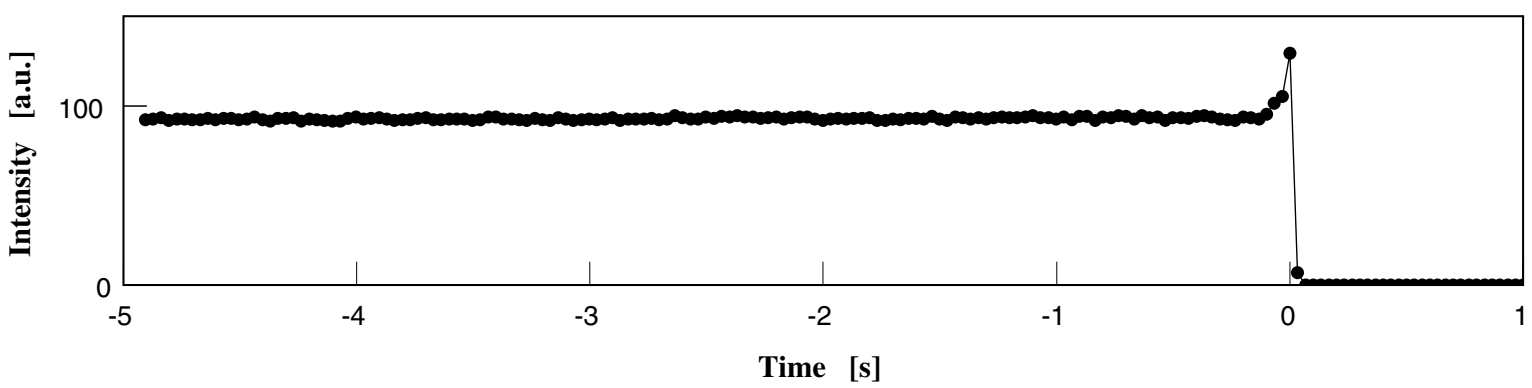

(g) Intensity of the pixels in the region inside the red square for each frame during the time period from $5 \mathrm{~s}$ before until $1 \mathrm{~s}$ after this cavity breakdown. The origin of the horizontal axis is the time at which this cavity breakdown occurred.

FIG. 14. Example of spot-type explosion events without a stable bright spot, accompanied by the sudden appearance of a new bright spot. (a)-(f) are snapshots, in chronological order, of the TEP recorded with TV camera 2 during the rf conditioning. The insets are magnified views of the region outlined by a red square. There was an explosion at 3 o'clock [indicated by the red square in (b)-(e)] at $V_{c}=0.80 \mathrm{MV}$; however, no clear bright spot was present in this region until 4 frames $(4 / 30 \mathrm{~s})$ before this cavity breakdown, as indicated by the red square in (a). A new small bright spot appeared in this region 3 frames $(0.1 \mathrm{~s})$ before this cavity breakdown, as indicated by the red square in (b). A clear spike was observed in the intensity of the region outlined by a red square at the moment of this cavity breakdown for four frames, as shown in $(\mathrm{g})$. 
disappearance of the bright spot, demonstrating that almost all the bright spots disappeared after an explosion occurred. This decrease in the number of explosion seeds (stable bright spots) is a significant component of rf conditioning, occurring in $20 \%$ or more of all cavity breakdown events. Figures 10 and 11 show snapshots of the FEP and TEP, respectively, taken in chronological order at $V_{c}=0.90 \mathrm{MV}$. The number of stable bright spots decreased over time, although some bright spots became brighter and some new bright spots appeared.
In Table IV, an event classified as the "spot-type explosion not originating from a stable bright spot" column (hereafter, a spot-type explosion event without a stable bright spot) is defined as a cavity breakdown event accompanied by a spot-type explosion at the position where no stable bright spot was observed until $2 \mathrm{~s}$ before the cavity breakdown. Spot-type explosion events with and without a stable bright spot are hereafter collectively called spot-type explosion events. Figure 12 shows an example of spot-type explosion events without

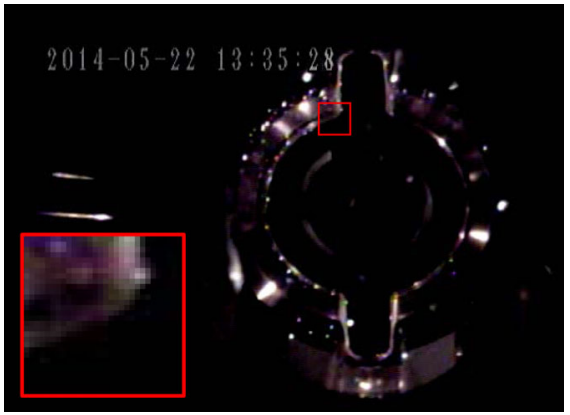

(a) 46 frames (46/30 s) before this cavity (b) 45 frames breakdown.

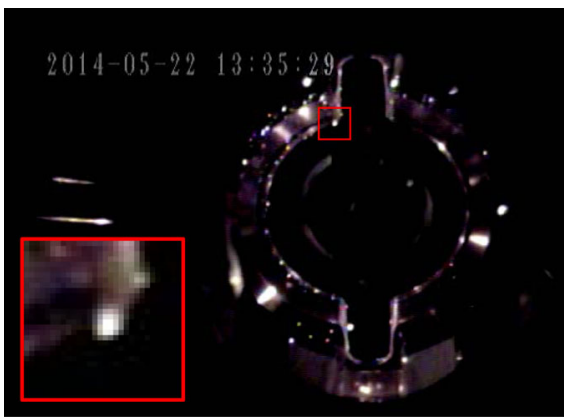

(d) 15 frames $(0.5 \mathrm{~s})$ before this cavity breakdown.

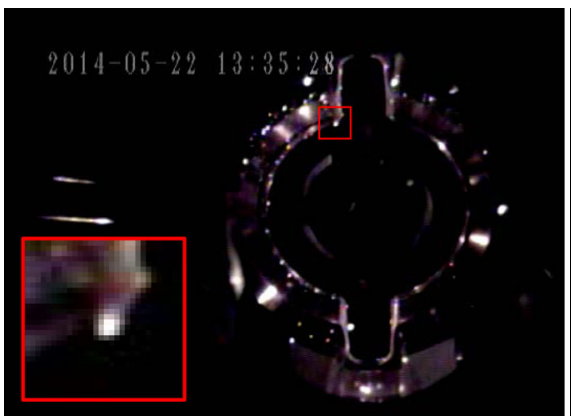

$(45 / 30 \mathrm{~s})$ before this cavity breakdown.

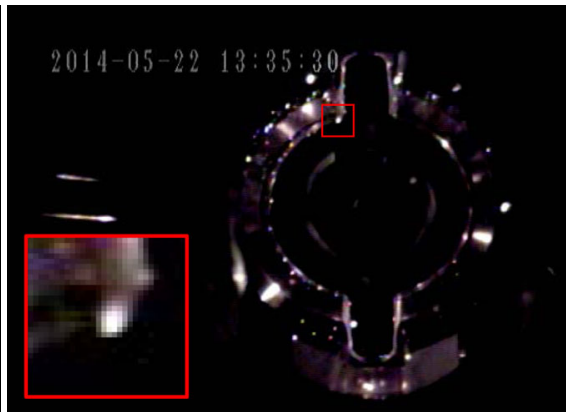

(e) 1 frame $(1 / 30 \mathrm{~s})$ before this cavity breakdown.

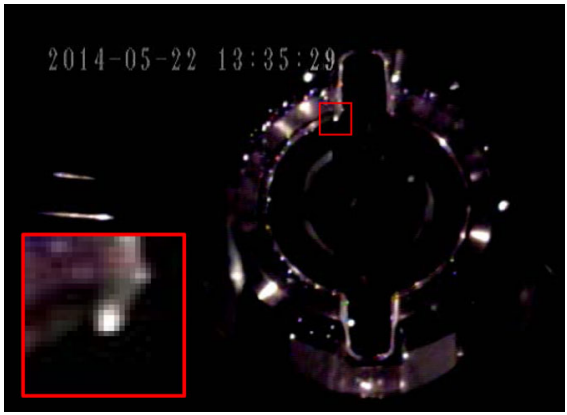

(c) 30 frames (1 s) before this cavity breakdown.

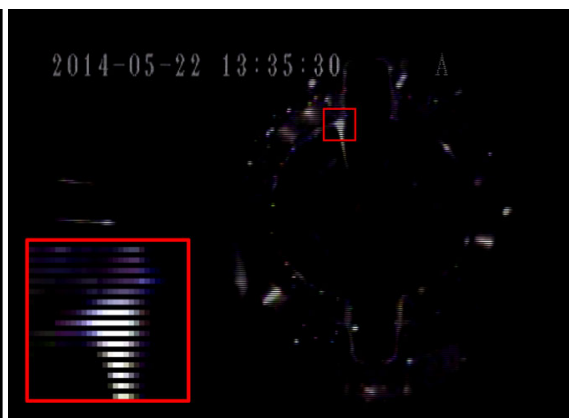

(f) At the moment of this cavity breakdown.

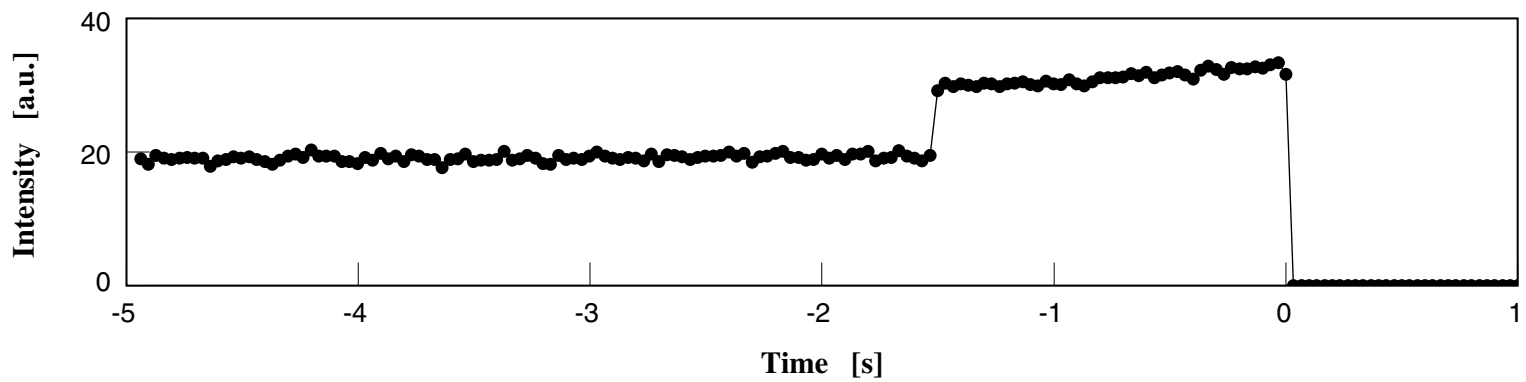

(g) Intensity of the pixels in the region inside the red square for each frame during the time period from $5 \mathrm{~s}$ before until $1 \mathrm{~s}$ after this cavity breakdown. The origin of the horizontal axis is the time at which this cavity breakdown occurred.

FIG. 15. Example of spot-type explosion events without a stable bright spot, accompanied by the sudden appearance of a new bright spot. (a)-(f) are snapshots, in chronological order, of the FEP recorded with TV camera 3 during the rf conditioning. The insets are magnified views of the region outlined by a red square. In this case, a new bright spot appeared at 11 o'clock 45 frames (1.5 s) before this cavity breakdown at $V_{c}=0.54 \mathrm{MV}$. A clear enhancement was observed in the intensity of the region outlined by a red square over the 46 frames, starting at the moment when the new bright spot appeared, as shown in $(\mathrm{g})$. 
a stable bright spot. It should be noted that, in a number of such events (on the order of several percentage points), a new bright spot suddenly appeared at the position of the explosion within $2 \mathrm{~s}$ before the cavity breakdown (Table VI). Figure 13 shows an example of such events, in which a new bright spot appeared one frame $(1 / 30 \mathrm{~s})$ before the cavity breakdown. Figure 14 shows another example of such events, in which a tiny new bright spot appeared three frames $(0.1 \mathrm{~s})$ before the cavity breakdown, then increased in intensity until the cavity breakdown occurred. Figure 15 shows a third example of such events, in which a new bright spot appeared 45 frames $(1.5 \mathrm{~s})$ before the cavity breakdown, then gradually increased in intensity until the cavity breakdown occurred. In these events, relevant microscopic dynamics (the generation, growth, and explosion of a bright spot, followed by cavity breakdown) occurred on a time scale

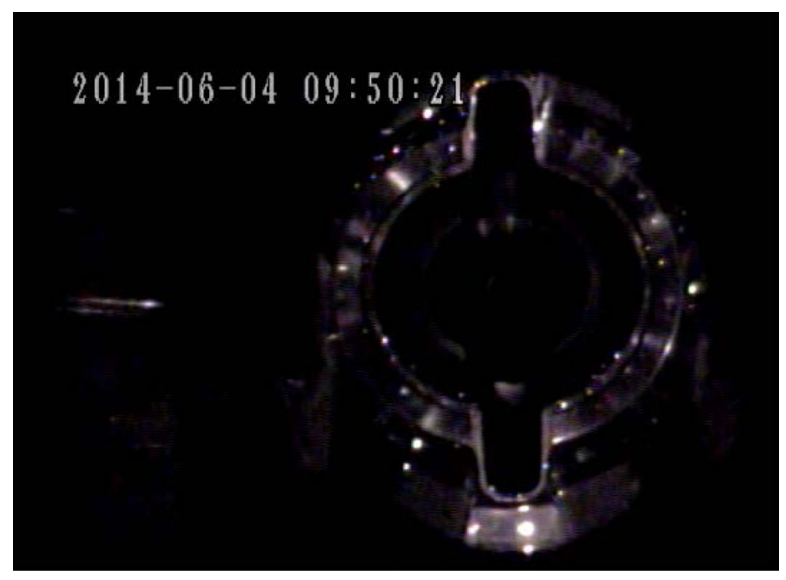

(a) 1 frame $(1 / 30 \mathrm{~s})$ before the cavity breakdown.

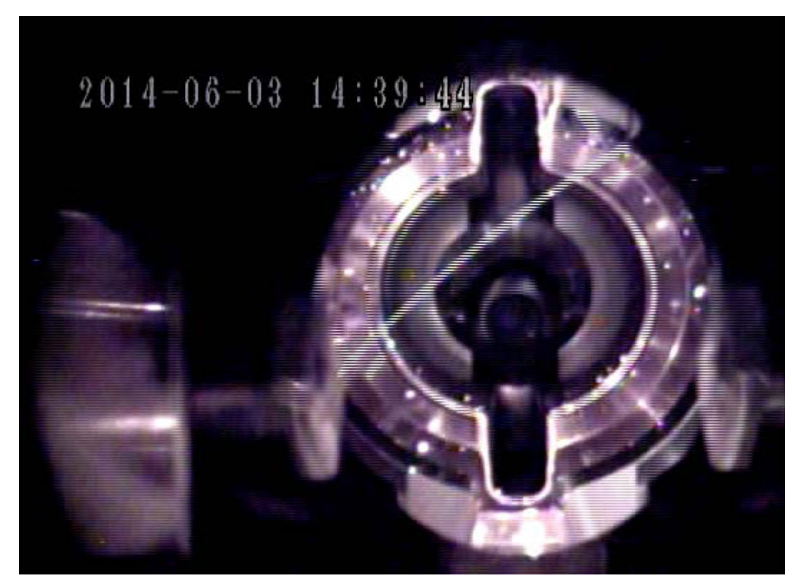

(c) Lightning captured with TV camera 3. of $1 \mathrm{~s}$ or less. It could be hypothesized that there is a mechanism underlying these dynamics that is common among all spot-type explosion events, regardless of whether a stable bright spot is present. This mechanism, if it exists, may be a hint to the cavity-breakdown trigger mechanism because spot-type explosion events are $60 \%$ or more of all cavity breakdown events.

Figure 16 shows examples of non-spot-type events, which are statistically insignificant. It is difficult to find any spots of abnormalities in such events because abnormalities are not localized.

\section{B. Nonuniformity of explosion spots}

Figure 17 shows the locations of the explosion spots on the end plates for the spot-type explosion events (132 points in total, sum of the numbers in the "spot-type explosion of a stable bright spot" and "spot-type explosion

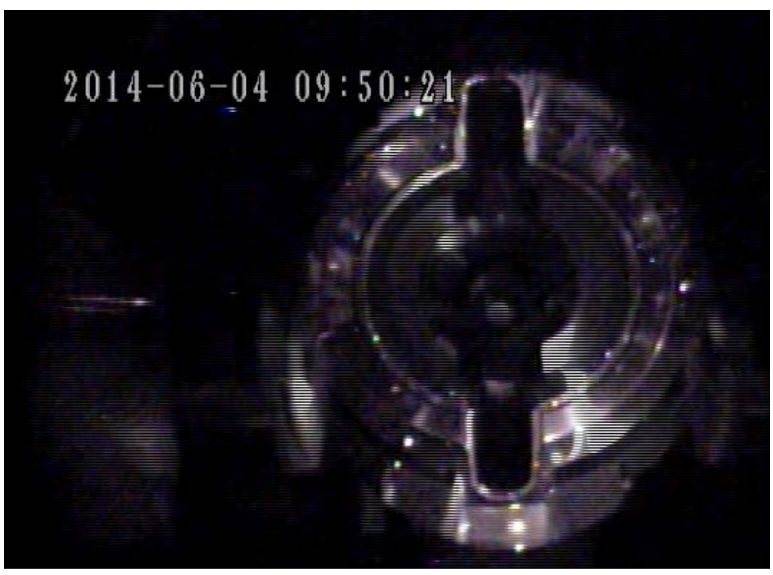

(b) Flash at the moment of the cavity breakdown.

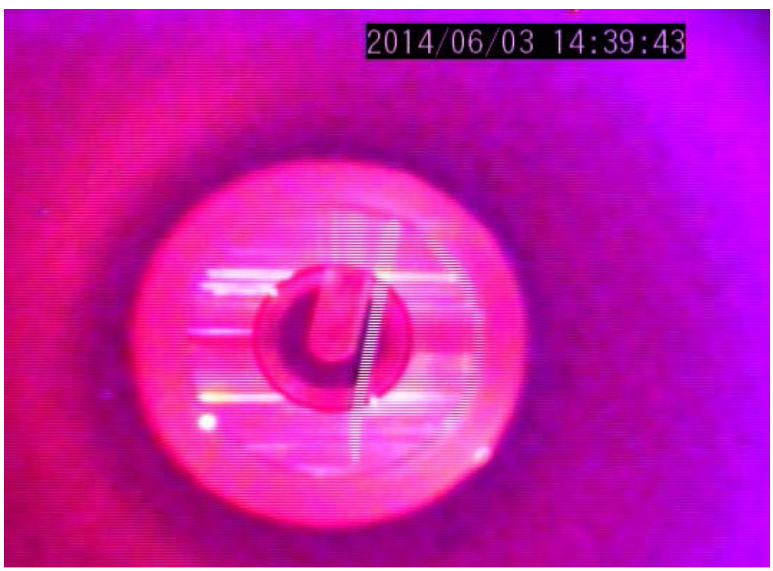

(d) Lightning captured with TV camera 1.

FIG. 16. Examples of non-spot-type breakdown events. (a) and (b) Cavity breakdown event at $V_{c}=0.76 \mathrm{MV}$ accompanied by a flash. This event is included in the "Non-spot-type flash" column in Table IV. (c) and (d) Snapshots 2/30 s before a cavity breakdown at $V_{c}=0.90 \mathrm{MV}$ accompanied only by lightning. No other phenomena were observed. This event is included in the "Non-spot-type lightning only" column in Table IV. 


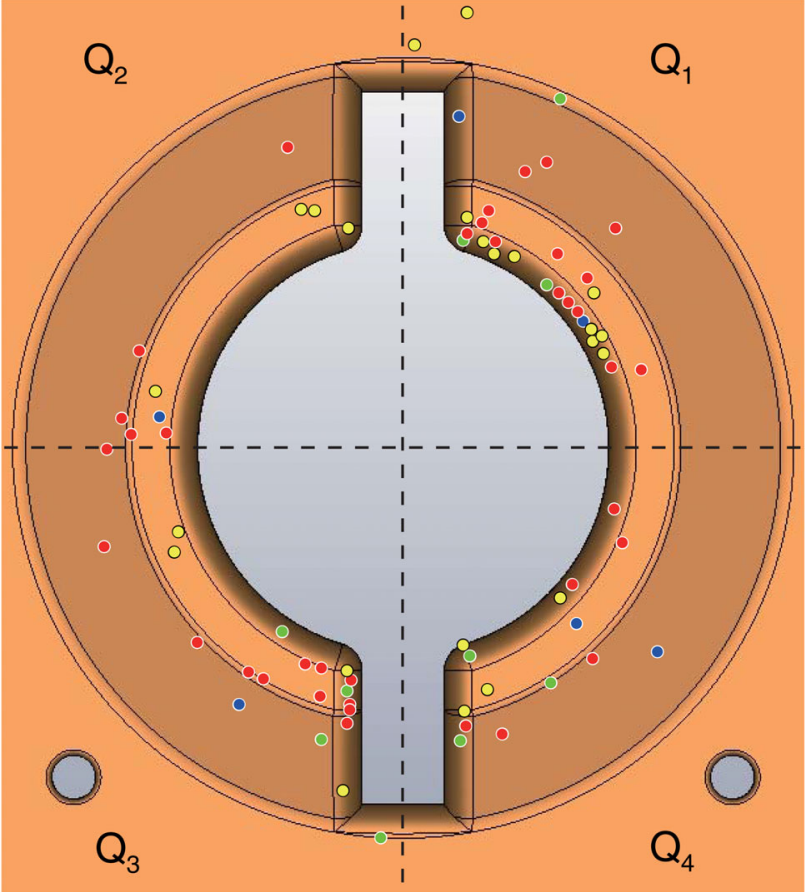

(a) FEP.

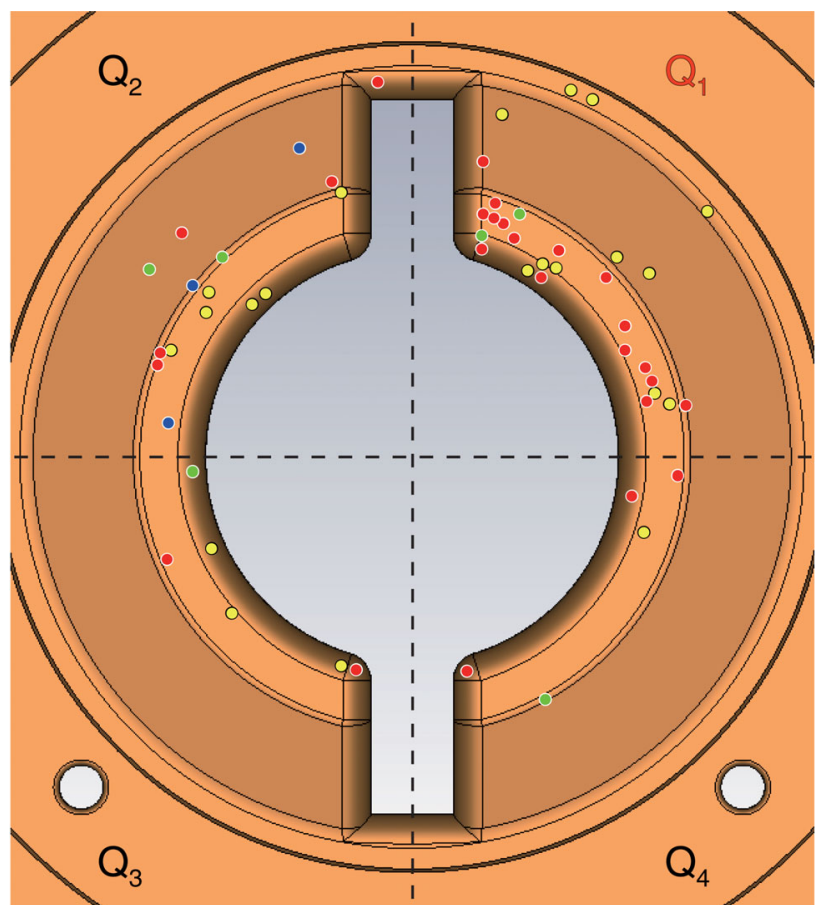

(b) TEP.

FIG. 17. Distribution of explosion spots for spot-type explosion events, shown as circles. The plot colors of blue, green, yellow, and red indicate cavity voltage ranges of $V_{c} \leq 0.40 \mathrm{MV}, 0.40 \mathrm{MV}<V_{c} \leq 0.60 \mathrm{MV}, \quad 0.60 \mathrm{MV}<V_{c} \leq 0.80 \mathrm{MV}$, and $0.80 \mathrm{MV}<V_{c} \leq 0.95 \mathrm{MV}$, respectively, at the moment of cavity breakdown. The small holes, two each for the FEP and TEP, are monitor ports for pickup antennas. The horizontal and vertical dashed lines define the quadrants used in Fig. 18. This partitioning yields the smallest possible sections having the same geometry. Quadrant $Q_{1}$ of the TEP, shown in red, is where the brazing filler metal leaked (Appendix B), and the effect of the leakage was at a visual maximum.

not originating from a stable bright spot" columns in Table IV). Here, the information obtained by TV camera 1 was often used to distinguish on which end plate (the FEP or TEP) the explosion occurred by detecting the brighter side when a cavity breakdown occurred. The fraction of the bright spot explosions that occurred on the FEP was $76 / 132=0.576_{-0.047}^{+0.046}$, where the binomial error is given with a $1 \sigma$ confidence interval. The statistical significance of the deviation from perfect uniformity (fraction of 0.5) is $1.65 \sigma$ (within the $90 \%$ confidence interval); therefore, the difference between the numbers of events occurring on the FEP and TEP $(76-56=20)$ is not statistically significant. However, nonuniformity can be seen in the azimuthal direction for both the FEP and TEP. Figure 18 quantitatively shows the nonuniformity; the maximum significance for the FEP is $2.66 \sigma$ at $\mathrm{Q}_{1}$ (outside the $99.2 \%$ confidence interval), which is not clearly significant. In contrast, the maximum significance for the TEP is $4.17 \sigma$ at $\mathrm{Q}_{1}$ (outside the $99.996 \%$ confidence interval), which is clearly significant. We demonstrated that this significant nonuniformity is correlated with the leakage of the brazing filler metal into the cavity from between the TEP and the barrel (Appendix B). No other causes of this nonuniformity were found in the production process of DR cavity No. 2. Therefore, we observed the effect of such leakage, which is not clearly seen in any other quantities, including the unloaded quality factor, rf conditioning speed, partial or total vacuum pressure, cavity breakdown rate, and radiation dose rate (or dark current).

\section{Field emission}

Field emission from the inner surfaces of the end plates leads to the generation of $\mathrm{x}$-rays when field-emitted electrons accelerate and impact inner surfaces. Therefore, the field emission can be estimated by measuring the radiation dose rate near the cavity. Figure 19(a) shows the radiation dose rates measured near DR cavities No. 0 , No. 1, and No. 2. The radiation dose rates of DR cavities No. 1 and No. 2 for cavity voltages $V_{c}$ ranging from 0.825 to $0.850 \mathrm{MV}$ are approximately one fifth that of DR cavity No. 0 ; this reduction in radiation dose rate is one of the results of electropolishing the end plates. The radiation dose rate of DR cavity No. 2 at cavity voltages $V_{c}$ ranging from 0.7 to $0.9 \mathrm{MV}$ is comparable to that of DR cavity No. 1. Figure 19(b) shows the radiation dose rate at $V_{c}=0.90 \mathrm{MV}$ for each day of the high-gradient test. The field emission level decreased during the $\mathrm{rf}$ 


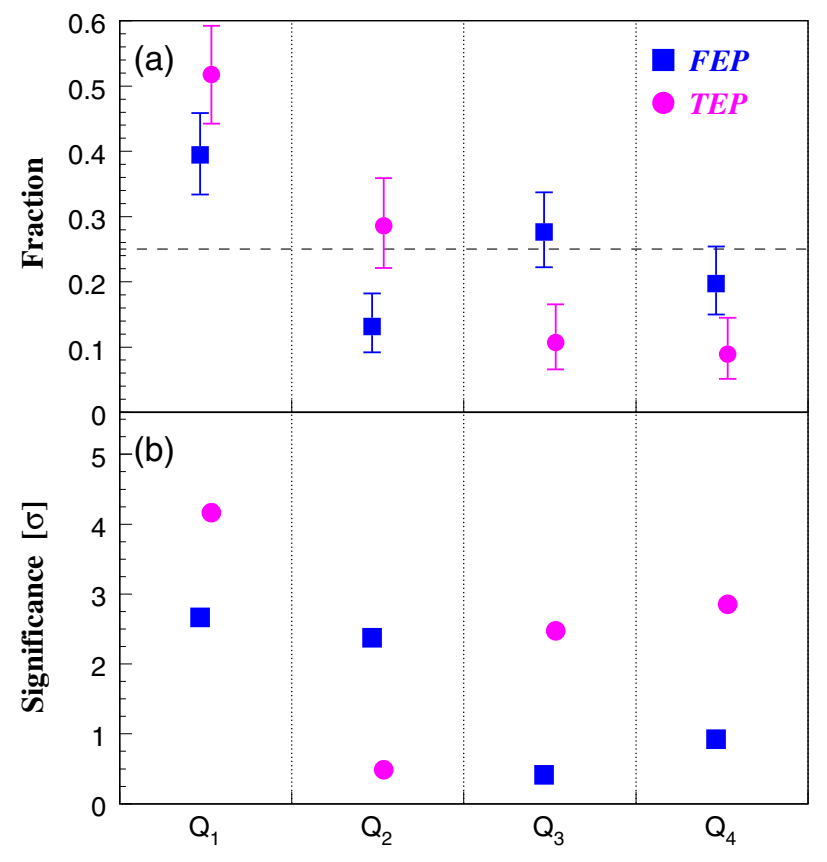

FIG. 18. (a) Fraction of spot-type explosion events with an explosion spot in each quadrant of the FEP (blue squares) and TEP (magenta circles), where the binomial errors are shown with a $1 \sigma$ confidence interval. Quadrants $Q_{1}, Q_{2}, Q_{3}$, and $Q_{4}$ are defined in Fig. 17. A fraction of 0.25 (perfect uniformity) is shown by the dashed line. (b) Statistical significance of the deviation from perfect uniformity.

conditioning then remained almost constant during the stability test. This is consistent with the data shown in Fig. 19(a), which demonstrates that the field emission level reached a minimum at the end of the rf conditioning. This means that the stable bright spots, which exploded when the cavity breakdown occurred, were not dominant continuous field emitters during the stability test before the explosion.

\section{CONCLUSIONS}

Through the breakdown study of the normalconducting 508.9-MHz continuous-wave single-cell cavity based on the direct observation method and statistical data analysis, we experimentally demonstrated the following conclusions. Clear bright spots were present on the end plates during high-gradient operation, most of which maintained their intensity with no significant effects on the high-gradient operation as long as they remained stable. Even after rf conditioning, such stable bright spots were observed during the stability test while a cavity voltage of $V_{c}=0.90 \mathrm{MV}$ was maintained. In $60 \%$ or more of the cavity breakdown events, a spot-type explosion occurred at the moment of cavity breakdown. That is, spot-type explosion events are the most frequently occurring type of cavity breakdown events. In $20 \%$ or more of the cavity

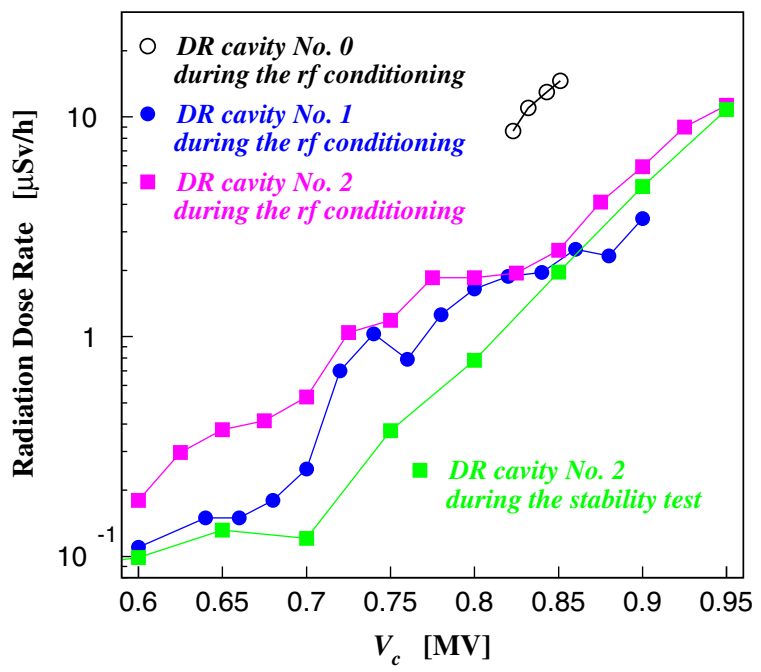

(a) Radiation dose rate as a function of $V_{c}$.

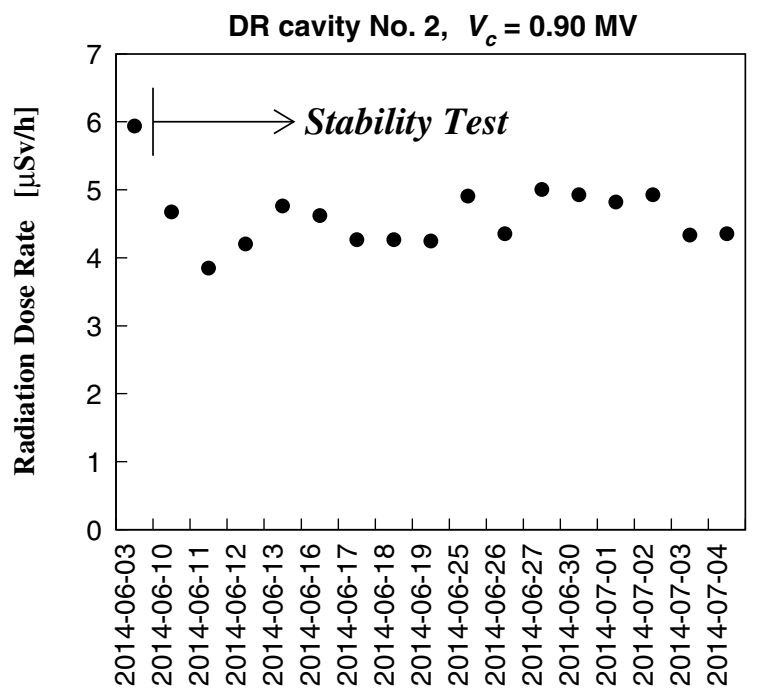

(b) Radiation dose rate as a function of time (day).

FIG. 19. Radiation dose rates measured approximately $2 \mathrm{~m}$ from the cavities in the radiation-controlled area. The background level was approximately $0.1 \mu \mathrm{Sv} / \mathrm{h}$.

breakdown events, a stable bright spot exploded and then disappeared. It has been verified that such bright spots were not dominant continuous field emitters before the explosion during the stability test. In approximately $3 \%$ of the cavity breakdown events, the sudden appearance of a new bright spot was observed within $2 \mathrm{~s}$ before the cavity breakdown, followed by the explosion of this bright spot when the cavity breakdown occurred. There was significant and unexpected nonuniformity in the distribution of the explosion spots on the TEP.

We also experimentally demonstrated that a decrease in the number of stable bright spots after an explosion is a significant component of rf conditioning. The observation 
of the sudden appearance of new bright spots shortly before cavity breakdowns (on the order of $1 \mathrm{~s}$ or less before cavity breakdowns) has stimulated our interest in the microscopic dynamics of the generation, growth, and explosion of bright spots and their correlation with rf conditioning effects and cavity breakdown rates because a possible mechanism underlying these dynamics may provide insight into the cavity-breakdown trigger mechanism. The direct observation method employed in this study may be a useful tool to quantitatively evaluate rf cavities, as demonstrated by the nonuniformity of the explosion spots.

This direct observation method can be extensively applied to more advanced breakdown studies using higherspecification cameras or detectors with higher speeds, higher resolution, and/or broader spectra. Such studies may illuminate the cavity-breakdown trigger mechanism.

\section{APPENDIX A: LIST OF ABBREVIATIONS}

The abbreviations used in this paper are listed in Table VII.

\section{APPENDIX B: DETAILS OF THE DR CAVITY}

Because the rf operating frequency of $508.9 \mathrm{MHz}$ is the same as that used for the normal-conducting rf cavity system ARES [21] (hereafter, simply referred to as ARES) used for the main rings of SuperKEKB, the basic structure of the DR cavity is the same as that of the accelerating cavity in ARES, where ARES is a three-cavity system stabilized with $\pi / 2$-mode operation, and consists of accelerating and energy-storage cavities with a coupling cavity between them. In particular, the DR cavity has a complete heavily HOM-damped structure with four HOM waveguides and two grooved beam pipes [22]. This HOM-damped structure is based on ARES, where the 32 sets of cavities of ARES had been successfully operated at the KEKB accelerator $[23,24]$ with extremely low cavity breakdown rates (less than one breakdown per cavity during 3 months of continuous operation at

TABLE VII. Abbreviations used in this paper.

\begin{tabular}{ll}
\hline \hline Abbreviation & Full letters \\
\hline ARES & $\begin{array}{l}\text { Accelerator resonantly coupled } \\
\text { with energy storage } \\
\text { Bright spot }\end{array}$ \\
BS & $\begin{array}{l}\text { Damping ring } \\
\text { DR }\end{array}$ \\
FEP & Fixed end plate \\
HOM & Higher order mode \\
IACS & International annealed copper standard \\
ICF & International conflat flange \\
rf & Radio frequency \\
TEP & Tuning end plate \\
TV & Television \\
\hline \hline
\end{tabular}

$\left.V_{c} \approx 0.5 \mathrm{MV}\right)$. We have adopted such HOM-damped structure because highly stable operation is required for the DR. Regarding the cavity voltage, we have chosen $V_{c}=0.80 \mathrm{MV}\left(E_{\mathrm{acc}}=3.1 \mathrm{MV} / \mathrm{m}\right)$ as the specification voltage (gradient) of the cavity; this cavity voltage is significantly higher than that of ARES $\left(V_{c}=0.50 \mathrm{MV}\right)$. Therefore, stable operation had to be demonstrated at such a high gradient.

The DR cavity has 12 ports in addition to the beam ports: two ICF203 ports in the horizontal direction (one is used as an input coupler, and the other is used for vacuum evacuation), one ICF203 port for a movable tuner (tuner port), one ICF70 port to view the tuner plunger, four ICF34 ports on the end plates for pickup antennas (monitor ports), and four HOM rectangular waveguides. With the exception of the tuner and monitor ports, the geometry of the vacuum region is symmetric with respect to both the horizontal and vertical planes including the beam axis. Additionally, with the exception of the monitor ports, HOM waveguides, and tuning bump on the TEP, it is also symmetric with respect to the vertical plane including the cavity center perpendicular to the beam axis (i.e., symmetric between the upstream and downstream halves).

In the final brazing of DR cavity No. 2, some of the brazing filler metal (BAg-8V) leaked from between the barrel and the TEP into the cavity, and sputtered on the TEP. Furthermore, some of the brazing filler metal that leaked into the cavity sputtered on the FEP. This did not occur during the fabrication of DR cavity No. 1. Therefore, before it was delivered, DR cavity No. 2 was vacuum baked for $15 \mathrm{~h}$ while maintaining a ribbon heater temperature of $100{ }^{\circ} \mathrm{C}$ with the ribbon heaters wrapped around the joints between the barrel and end plates. This temperature of $100^{\circ} \mathrm{C}$ is higher than the maximum outer surface temperature of the copper body of the cavity during high-gradient operation $\left(60^{\circ} \mathrm{C}\right)$. Before, after, and during the baking, it was confirmed using a $Q$ mass spectrometer that no gas components were present other than those observed for DR cavity No. 1, and that no spikes or abnormal increases in the vacuum pressure occurred while the vacuum pressure in the cavity was kept below $3.5 \times 10^{-5} \mathrm{~Pa}$. Finally, DR cavity No. 2 passed a typical vacuum leak test with a background level lower than $1.3 \times 10^{-10} \mathrm{~Pa} \mathrm{~m}^{3} / \mathrm{s}$.

Other details of the DR cavity are given in [12].

[1] W. Wuensch et al., Experience operating an X-band highpower test stand at CERN, in Proceedings of the 5th International Particle Accelerator Conference (IPAC2014), Dresden, Germany (2014), Paper ID: WEPME016, http://accelconf.web.cern.ch/AccelConf/IPAC2014/papers/ wepme016.pdf.

[2] J. Norem, V. Wu, A. Moretti, M. Popovic, Z. Qian, L. Ducas, Y. Torun, and N. Solomey, Dark current, breakdown, and magnetic field effects in a multicell, 
805 MHz cavity, Phys. Rev. ST Accel. Beams 6, 072001 (2003).

[3] T. Higo et al., Various observables of TW accelerator structures operating in $100 \mathrm{MV} / \mathrm{m}$ or higher at X-band facility, Nextef of KEK, in Proceedings of the 1st International Particle Accelerator Conference (IPAC'10), Kyoto, Japan (JACoW, Geneva, 2010), THPEA012, http://accelconf.web .cern.ch/AccelConf/IPAC10/papers/thpea012.pdf.

[4] S. Matsumoto, T. Abe, Y. Higashi, T. Higo, and Y. Du, High gradient test at Nextef and high-power long-term operation of devices, Nucl. Instrum. Methods Phys. Res., Sect. A 657, 160 (2011).

[5] A. Grudiev, S. Calatroni, and W. Wuensch, New local field quantity describing the high gradient limit of accelerating structures, Phys. Rev. ST Accel. Beams 12, 102001 (2009); 14, 099902(E) (2011).

[6] V. Dolgashev, S. Tantawi, Y. Higashi, and B. Spataro, Geometric dependence of radio-frequency breakdown in normal conducting accelerating structures, Appl. Phys. Lett. 97, 171501 (2010).

[7] F. Wang, C. Adolphsen, and C. Nantista, Performance limiting effects in X-band accelerators, Phys. Rev. ST Accel. Beams 14, 010401 (2011); 15, 120402 (2012).

[8] L. R. Grisham, A. Von Halle, A. F. Carpe, G. Rossi, K. R. Gilton, E.D. McBride, E.P. Gilson, A. Stepanov, and T.N. Stevenson, Studies of electrical breakdown processes across vacuum gaps between metallic electrodes, Nucl. Instrum. Methods Phys. Res., Sect. A 733, 168 (2014).

[9] T. Otsuka, Y. Yamano, S. Kobayashi, T. Higo, N. Toge, K. Takata, Y. Higashi, Y. Saito, and N. Hitomi, Vacuum electrical breakdown characteristics of oxygen-free copper electrodes processed by precision machining, in Proceedings of the 20th International Symposium on Discharges and Electrical Insulation in Vacuum (IEEE, New York, 2002), p. 556, http://ieeexplore.ieee.org/ document/1027432/.

[10] N. Shipman, Ph.D. thesis, Manchester U., 2014, http:// inspirehep.net/record/1381397/files/CERN-THESIS-2014278.pdf.

[11] D. P. Pritzkau and R. H. Siemann, Experimental study of rf pulsed heating on oxygen free electronic copper, Phys. Rev. ST Accel. Beams 5, 112002 (2002).

[12] T. Abe et al., SuperKEKB design report to be published in KEK report, http://www-superkekb.kek.jp/documents.html.

[13] R. A. Rimmer, M. Allen, K. Fant, A. Hill, M. Hoyt, J. Judkins, J. Saba, H. Schwarz, and M. Franks, High-power testing of the first PEP-II rf cavity, Conf. Proc. C960610, 2032 (1996).

[14] J. D. Fox (private communication).

[15] R. Dowd, G. LeBlanc, and K. Zingre, Commissioning and operation of the $500 \mathrm{MHz}$ storage ring rf system for the Australian synchrotron, Nucl. Instrum. Methods Phys. Res., Sect. A 592, 224 (2008).

[16] R. Dowd (private communication).

[17] T. Abe, T. Kageyama, H. Sakai, Y. Takeuchi, and K. Yoshino, High power testing of the rf accelerating cavity for the positron damping ring at SuperKEKB, in Proceedings of the 10th Annual Meeting of Particle Accelerator Society of Japan (Particle Accelerator Society of Japan, Tokyo, 2013), Paper ID: SAP057, http://www.pasj.jp/ web_publish/pasj10/proceedings/PDF/SAP0/SAP057.pdf.

[18] T. Abe, T. Kageyama, H. Sakai, Y. Takeuchi, and K. Yoshino, Multipactoring suppression by fine grooving of conductor surfaces of coaxial-line input couplers for high beam current storage rings, Phys. Rev. ST Accel. Beams 13, 102001 (2010).

[19] Note1, the brightness (contrast) of all the video images shown in this paper, except for Figs. 16(a) and 16(b), has been reduced (enhanced) to more clearly show bright spots by using the following command from the ImageMagick software suite: convert brightness-contrast $-20 \times 40$. The contrast of the images in Figs. 16(a) and 16(b) has been enhanced by using the following command: convert brightness-contrast $0 \times 30$.

[20] T. Abe, T. Kageyama, H. Sakai, Y. Takeuchi, and K. Yoshino, Test results on rf accelerating cavities for the positron damping ring at SuperKEKB, in Proceedings of the 11th Annual Meeting of Particle Accelerator Society of Japan (Particle Accelerator Society of Japan, Tokyo, 2014), Paper ID: SAP050, http://www.pasj.jp/web_ publish/pasj2014/proceedings/PDF/SAP0/SAP050.pdf.

[21] T. Kageyama et al., Report No. KEK-PREPRINT-98-45, 1998, https://lib-extopc.kek.jp/preprints/PDF/1998/9827/ 9827045.pdf.

[22] T. Kageyama, Report No. KEK-PREPRINT-91-133, 1991, https://lib-extopc.kek.jp/preprints/PDF/1991/9127/ 9127133.pdf.

[23] T. Abe et al., Achievements of KEKB, Prog. Theor. Exp. Phys. 2013, 03A001 (2013).

[24] T. Abe et al., Performance and operation results of the $\mathrm{rf}$ systems at the KEK B-Factory, Prog. Theor. Exp. Phys. 2013, 03A006 (2013). 